\title{
On the Similarity of Lower-Stratospheric Potential Vorticity Dipoles above Tropical and Midlatitude Deep Convection
}

\author{
MatThew H. Hitchman AND Shellie M. Rowe \\ Department of Atmospheric and Oceanic Sciences, University of Wisconsin-Madison, Madison, Wisconsin
}

(Manuscript received 13 August 2016, in final form 20 May 2017)

\begin{abstract}
Simulations of the effects of deep convection on the structure of potential vorticity (PV) in the upper troposphere and lower stratosphere (UTLS) have shown that a common signature in the presence of ambient horizontal vorticity is a horizontal PV dipole. Here, the relationship between convection and PV structures in the UTLS in Tropical Cyclone Talas and the extratropical "Super Tuesday" cyclone is investigated with the University of Wisconsin Nonhydrostatic Modeling System (UWNMS). Dipoles of potential temperature in the UTLS are interpreted as an upward deflection of the ambient flow over the updraft (cold), followed by subsidence in its lee (warm), aligned with the wind direction. PV dipoles larger than $\pm 20 \mathrm{PV}$ units $(1 \mathrm{PVU}=$ $10^{-6} \mathrm{~K} \mathrm{~kg}^{-1} \mathrm{~m}^{2} \mathrm{~s}^{-1}$ ) are identified, with typical vertical and horizontal extents of $\sim 3$ and $\sim 200 \mathrm{~km}$, respectively, and lifetimes up to $12 \mathrm{~h}$. Confirming the findings of Chagnon and Gray, it is found that horizontal PV dipoles are related to vortex tilting, where horizontally oriented vorticity associated with vertical shear of the ambient wind is bent into a horseshoe shape by the updraft, yielding a PV dipole. This suggests that theta dipoles are perpendicular to PV dipoles and that "low PV lies to the left of the wind shear," or, in the case of tropical cyclones, "low PV lies radially outward." Mesoscale jets occur between the dipoles, which oppose the ambient anticyclonic flow. During the extratropical transition of Talas, convective PV anomalies evolved under synoptic-scale deformation into a pair of PV streamers, which modified the midlatitude westerly jet.
\end{abstract}

\section{Introduction}

Recent studies of simulations of deep convection (e.g., Chagnon and Gray 2009) and of balanced vortex dipoles (e.g., Snyder et al. 2007, 2009; Wang et al. 2009; Hernandez-Duenas 2014) have shown that deep convection impinging on the base of the stratosphere can generate significant horizontal dipoles of potential vorticity (PV) in the upper troposphere and lower stratosphere (UTLS), each of which necessarily has a horizontal jet in between. Here we diagnose simulations of tropical cyclone Talas and the extratropical cyclone Super Tuesday storm using the University of Wisconsin Nonhydrostatic Modeling System (UWNMS) to study PV structures in the UTLS over clusters of deep convection. How can large positive and negative PV

Supplemental information related to this paper is available at the Journals Online website: http://dx.doi.org/10.1175/ JAS-D-16-0239.s1.

Corresponding author: Matthew H. Hitchman, matt@aos. wisc.edu anomalies be created in the UTLS? Our results confirm the reasoning of Chagnon and Gray (2009) that ambient horizontal vorticity can be modified by an updraft to create horizontal PV dipoles. The current literature suggests a range of points of view with regard to the physical nature and mathematical treatment of $\mathrm{PV}$ conservation. Here the role of the tilting/twisting term in the budgets for absolute vorticity and PV will be further explored.

Chagnon and Gray (2009) studied horizontal PV dipoles of magnitude $10 \mathrm{PV}$ units $\left(1 \mathrm{PVU}=10^{-6} \mathrm{~K} \mathrm{~kg}^{-1} \mathrm{~m}^{2} \mathrm{~s}^{-1}\right)$ near the top of deep convection in idealized and detailed numerical simulations and showed that they can be created by vertical variation in latent heating plus vortex tilting. They found that at coarser resolution $(10 \mathrm{~km})$ the vertical PV dipole was dominant, while at $1-\mathrm{km}$ resolution, the tilting/twisting process was more evident, with more robust horizontal PV dipoles. Their results will be described further in the next section. Kilroy et al. (2014) included background rotation in a wind shear profile and found that simulated convection produced a vertical vorticity anomaly associated with the tilting of horizontal vorticity. They found a second, oppositely signed vertical 
vorticity anomaly generated by the tilting of the ambient horizontal vorticity by the convective downdraft. Consistent with these results, Conzemius and Montgomery (2009) used trajectory analysis in simulations to show that convective regions have strong PV anomalies of negative and positive sign. Additionally Weijenborg et al. (2015) used a nonhydrostatic numerical weather prediction model to investigate two specific cases to better understand quantities such as wind velocity associated with PV anomalies and storm updrafts. Deng et al. (2012) created an idealized three-dimensional Boussinesq model to study the formation of vertical hot towers and discovered that, when vertical shear is sufficiently strong, individual hot towers possess a vorticity dipole. A primary goal of the present work is to explore the relationship of convective PV dipole structures to ambient shear in the UTLS with respect to vortex tilting by convective updrafts and compare them in tropical and extratropical cyclones.

Snyder et al. (2007) investigated vortex dipoles associated with a convective updraft in rotating stratified fluid using idealized numerical simulations. They found long-lived balanced vortex dipoles with an outflow jet between each dipole in the UTLS. They also found inertia-gravity waves embedded within and stationary with respect to the dipole. Snyder et al. (2009) showed that these gravity waves are generated as a forced linear response to the balanced flow. Using geostrophic initial conditions taken from the surface-trapped quasigeostrophic dipole of Muraki and Snyder (2007), they found that the dipole undergoes a transient period in which waves radiate upward and away from the dipole. Wang et al. (2009) showed that inertia-gravity waves are nearly phase locked with respect to the jet, suggesting spontaneous emission. Wang et al. (2010) showed that the jet structure between the dipole tends to select for dominant gravity wave structures through the wave capture mechanism. Hernandez-Duenas et al. (2014) compared the evolution of quasigeostrophic and Boussinesq PV dipoles and found that emission of gravity waves is essential for creating a balanced state. Plougonven and Zhang (2014) provide a useful overview of gravity wave emission from jets and fronts and suggest that continuous emission can occur without balance being achieved. Our results also show that gravity waves are integral to the formation of PV dipoles. However, further analysis is warranted, and discussion of these results will be published in a separate manuscript.

Here, $\mathrm{PV}$ dipoles are investigated using the local vertical component of absolute vorticity, $\zeta_{3}+f$, Ertel's potential vorticity $\left[\mathrm{PV}=(1 / \rho)(\partial \theta / \partial z)\left(\zeta_{3}+f\right)\right]$, and equivalent potential vorticity $\left[\mathrm{EPV}=(1 / \rho)\left(\partial \theta_{e} / \partial z\right)\left(\zeta_{3}+f\right)\right]$, where $\zeta_{3}=\partial v / \partial x-\partial u / \partial y$ is the vertical component of relative vorticity, $f=2 \Omega \sin \varphi$ is the vertical component of Earth's vorticity (with Earth's rotation rate $\Omega$ and latitude $\varphi$ ), $\theta_{e}=\theta \exp \left(L w_{s} / c_{p} T\right)$ is equivalent potential temperature, $\theta$ is potential temperature, $L$ is the latent heat of condensation, $w_{s}$ is saturation water vapor mixing ratio, $c_{p}$ is specific heat at constant pressure, $T$ is temperature, and $\rho$ is density. The conceptual benefit of using EPV is that the inclusion of moisture allows tracing air with certain EPV to its origins in the boundary layer. It allows for understanding the production of vertical dipoles of PV due to latent heating causing vortex stretching in the lower troposphere and vortex shrinking in the upper troposphere. As water vapor content becomes small and $\theta_{e} \rightarrow \theta$, EPV asymptotically approaches PV in the UTLS (Rowe and Hitchman 2015, 2016). In the UTLS, regions where absolute vorticity, PV, and EPV are negative (indicating inertial instability) coincide almost exactly. Profiles of PV and EPV will be shown to be nearly identical in convective updrafts and in lower-stratospheric PV anomalies. In this work, a negative (positive) region will be referred to interchangeably as a negative (positive) anomaly of absolute vorticity, PV, or EPV. Budgets for absolute vorticity, linearized Boussinesq PV, and Ertel's PV will be discussed in the next section. We investigate the PV dipole structures in the UTLS over deep convection for Tropical Cyclone Talas in September 2011 and compare it with structures over convection associated with the Super Tuesday midlatitude cyclone of February 2008.

PV dynamics associated with midlatitude cyclones have been studied extensively for over a century (e.g., Bjerknes 1898; Eady 1949; Kleinschmidt 1950a,b, 1955; Eliassen and Kleinschmidt 1957; Hoskins et al. 1985). More recently, Chagnon et al. (2013), using a mesoscale model simulation of a cyclone in the North Atlantic, diagnosed a synoptic-scale layered horizontal dipole pattern of PV near the tropopause, which they attributed to radiative and latent heating processes in uppertropospheric cloud layers. PV also plays a significant role in tropical cyclone development (Wu and Emanuel 1995; Wang and Holland 1996; Shapiro and Franklin 1995). When a tropical cyclone begins to interact with a midlatitude environment, the characteristics of the cyclone can change dramatically (Palmen 1958; Muramatsu 1985; Foley and Hanstrum 1994). Thorpe (1986) studied the tangential wind associated with an isolated, circular upper-tropospheric positive PV anomaly and found that, if the anomaly did not change size or shape, the cyclone would weaken upon interaction with its environment. Jones et al. (2003) studied the tracks of all tropical cyclones that recurved toward the pole and underwent tropical transition during the 30 -yr period 1970-99. They reported that, in satellite imagery, the 
inner core of the tropical cyclone loses its symmetric appearance and gradually takes on the asymmetry of an extratropical cyclone during extratropical transition. During the extratropical transition of Tropical Cyclone Talas, and in the case of the Super Tuesday midlatitude cyclone, $\mathrm{PV}$ anomalies produced by deep convection in the UTLS are organized by horizontal deformation into elongated PV streamers which modify midlatitude jet structures.

Wind shear plays a significant role in tropical cyclone intensification, weakening, and maintenance. Zhang and Tao (2013) studied the effects of vertical wind shear and flow asymmetry on the intensity of a tropical cyclone during different stages of its life cycle. In the present work, wind shear in the upper levels and the absolute vorticity within convection will be examined as it relates to the formation of horizontal EPV dipoles in the UTLS and the extratropical transition of the aggregated dynamical system.

This work will feature the formation of an extensive, long-lasting PV dipole in the UTLS during Talas, located over the region of most intense rainfall, the Kii Peninsula, a southeastward extension of the Kansai region of Honshu, Japan. These dipoles will be compared with a structures associated with deep convection in the midlatitude Super Tuesday storm. In section 2, two fundamental "mental models" for the origin of theta dipoles and PV dipoles in the UTLS are presented as a means of focusing discussion throughout the rest of the paper. Section 3 describes the UWNMS simulations and auxiliary data used in this work. EPV dipoles in Tropical Cyclone Talas are investigated in section 4 , focusing on dipole formation, their structure and orientation with respect to ambient shear, the bias in outflow from the negative member of the dipole, and the formation of PV streamers during extratropical transition. Section 4 concludes with a brief exploration of the effects of differing model resolution on PV dipoles. Section 5 discusses PV dipoles in the midlatitude cyclone Super Tuesday storm of 6-8 February 2008 (Rowe and Hitchman 2015, 2016), with a comparison to those seen above Talas. Conclusions are given in section 6.

\section{Theta dipoles and PV dipoles}

Numerical simulations of deep convection in the UWNMS often exhibit horizontal dipoles in both potential temperature and potential vorticity in the UTLS. When convection in the model does not reach the tropopause, PV dipoles are not observed. Here, two mental models are introduced, which may provide insight in diagnosing convectively generated potential temperature

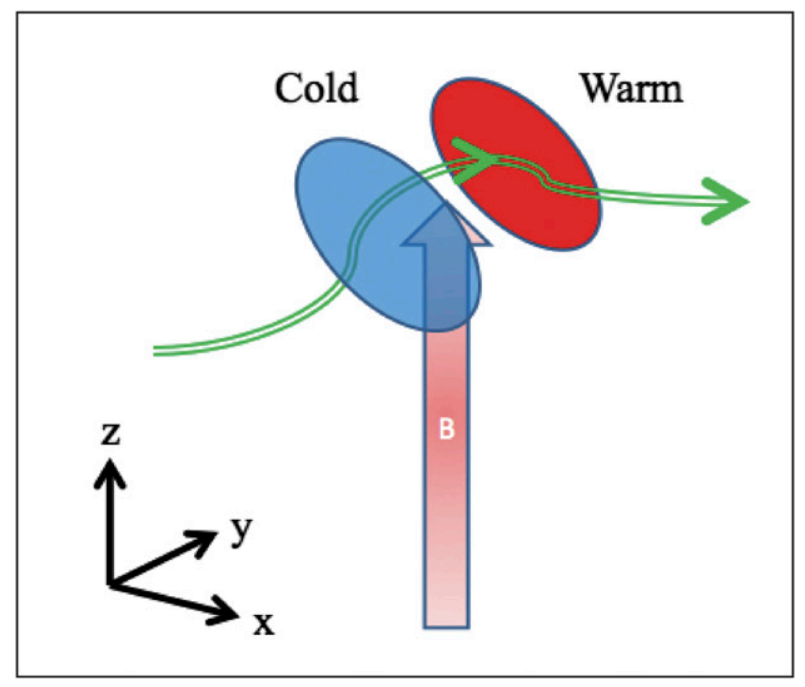

FIG. 1. Oblique view of an idealized theta dipole caused by a buoyant updraft $B$ deflecting horizontal flow in the UTLS, upward, ahead of, and over the updraft, creating the cold anomaly, with subsidence beyond the updraft creating the warm anomaly. This is similar to the deformation of the water surface in streamflow over a rock. This process can excite gravity waves, which extend the cold-warm anomaly upward and downward into the core of the thunderstorm.

and PV dipoles in tropical and extratropical cyclones. In the presence of weak horizontal motion in the UTLS, a convective updraft will generate a "theta monopole" of a cold dome centered on the updraft. But an updraft that penetrates into strong horizontal flow in the UTLS can create a "theta dipole" by deflecting part of the flow upward, yielding a cold anomaly, with subsidence in the lee of the updraft creating a warm anomaly (Fig. 1). This behavior is similar to streamflow over a rock or over an isolated mountain [e.g., Fig. 13.3 of Markowski and Richardson (2010)]. It is an integral feature of both the Super Tuesday deep convection outbreak and the behavior of convection in Tropical Cyclone Talas. It is intimately related to the formation of gravity waves and related phenomena, such as "jumping cirrus" (Wang 2004).

Chagnon and Gray (2009) showed that horizontal PV dipoles in the UTLS can be explained by tilting of horizontal vorticity into the vertical. Contemporary conceptual models of tornadogenesis include the idea of an updraft bending horizontally oriented vorticity, associated with downdraft outflow, into a horseshoe shape, which implies the creation of a dipole or pair of vertically oriented vortices (Markowski and Richardson 2010). First, the budgets of linearized Boussinesq PV, absolute vorticity, and Ertel's PV are discussed. Then we explore a mental model for PV dipole production by updrafts tilting horizontal vorticity into the vertical, 
in situations ranging from northeastward UTLS shear in a midlatitude cyclone to azimuthal shear in a tropical cyclone.

Chagnon and Gray (2009) used the linearized Boussinesq equations, which retain buoyant acceleration due to latent heat release $B$ in the presence of linear shear $\Lambda=d v / d z$, to show that the tendency of pseudopotential vorticity $q=\zeta_{3}+f(\partial / \partial z)\left(B / N^{2}\right)-\Lambda(\partial / \partial x)\left(B / N^{2}\right)$, is

$$
\frac{\partial q}{\partial t}=\left(f \frac{\partial}{\partial z}-\Lambda \frac{\partial}{\partial x}\right)\left(\frac{B}{N^{2}}\right),
$$

where $f$ is the Coriolis parameter and $N$ is the buoyancy frequency. The two forcing terms are called "latent stretching" and "latent tilting." In the presence of deep convection, the latent stretching term can create a vertical PV dipole centered on the level of maximum latent heating. Below that level, buoyant acceleration increases with altitude, increasing cyclonic $q$ by vortex stretching, while above that level $B$ decreases with altitude, leading to vortex compression and production of anticyclonic $q$ [cf. Schemm et al. (2013) regarding PV anomaly production in the warm-upglide pathway of extratropical cyclones]. Chagnon and Gray (2009) showed that the latent tilting term can alter this fundamental vertical dipole production, creating a horizontal PV dipole in the UTLS over convective updrafts (cf. their Figs. 1 and 9).

The strong correlation between vertical motion and latent heat release in convection, such that $w \propto B$, provides a conceptual bridge between Chagnon and Gray's work and the classical tilting term in the vorticity equation in Holton (2006). Taking the curl of the horizontal equations of motion, scaled for synoptic flows, the rate of change of absolute vorticity following horizontal motion is given by

$$
\begin{aligned}
\frac{D}{D t}\left(\zeta_{3}+f\right)= & -\left(\zeta_{3}+f\right)\left(\frac{\partial u}{\partial x}+\frac{\partial v}{\partial y}\right)-\left(\frac{\partial w}{\partial x} \frac{\partial v}{\partial z}-\frac{\partial w}{\partial y} \frac{\partial u}{\partial z}\right) \\
& +\frac{1}{\rho^{2}}\left(\frac{\partial \rho}{\partial x} \frac{\partial p}{\partial y}-\frac{\partial \rho}{\partial y} \frac{\partial p}{\partial x}\right),
\end{aligned}
$$

where the first term involves vortex stretching (compression) associated with horizontal divergence (convergence), the third term is the solenoidal or baroclinic term, and the second term is the tilting term, of primary interest in our diagnosis. As a comparison with the geometrical configurations in Chagnon and Gray (2009), in the case of pure poleward flow increasing with altitude, the second term in (2) reduces to $-(\partial w / \partial x)(\partial v / \partial z) \propto-(\partial B / \partial x) \Lambda$, which is the tilting term in (1).
Andrews et al. (1987) showed that the primitive equation version of Ertel's PV in log-pressure altitude coordinates [their (3.1.4)] and its conservation [their (3.1.5)] are

$$
\begin{aligned}
P= & \frac{1}{\rho} \frac{\partial \theta}{\partial z}\left(\zeta_{3}+f\right)+\frac{1}{\rho}\left(-\frac{\partial \theta}{\partial x} \frac{\partial v}{\partial z}+\frac{\partial \theta}{\partial y} \frac{\partial u}{\partial z}\right) \text { and } \\
\frac{d P}{d t}= & \frac{1}{\rho a \cos \varphi}\left[-\frac{\partial(X \cos \varphi, \theta)}{\partial(\varphi, z)}+\frac{\partial(Y, \theta)}{\partial(\lambda, z)}\right. \\
& \left.-\frac{\partial(Q, v)}{\partial(\lambda, z)}+\frac{\partial(Q, m)}{\partial(\varphi, z)}\right]
\end{aligned}
$$

where $Q$ is diabatic heating, $m$ is angular momentum per unit mass divided by $a$, and $X$ and $Y$ are subgrid-scale mechanical forcing acting on $u$ and $v$, respectively. In isentropic coordinates the PV conservation equation becomes [their (3.8.5)]

$$
\begin{aligned}
\frac{D P}{D t}= & \frac{1}{\sigma a \cos \varphi}\left(-\frac{\partial X \cos \varphi}{\partial \varphi}+\frac{\partial Y}{\partial \lambda}-\frac{\partial Q}{\partial \lambda} \frac{\partial v}{\partial \theta}+\frac{\partial Q}{\partial \varphi} \frac{\partial u}{\partial \theta} \cos \varphi\right) \\
& +P \frac{\partial Q}{\partial \theta}-Q \frac{\partial P}{\partial \theta},
\end{aligned}
$$

where $\sigma$ is isentropic density. Equations (4) and (5) show that if there is no net heating or mechanical forcing, $P$ is conserved following the motion. Diabatic and frictional processes can change $P$ following the motion. "Latent stretching terms" are found on the right-hand sides of (1), (4), and (5), and this process can contribute toward the stretching term in (2). To the extent that $w \propto Q$, tilting terms may be found on the rhs of (4) and (5). This further suggests that horizontal anomalies of $\mathrm{PV}$ require latent heating or friction to generate them in the first place.

In the UWNMS there is no explicit equation for conservation of PV. Instead, PV is calculated in altitude coordinates at each time step as a function of changing winds and potential temperature. Therefore, any diabatic heating processes or tilting processes that affect $u, v, w$, and $\theta$ will change PV. Although it may seem unusual that large PV anomalies can arise in the UTLS in numerical models, spatial variations in diabatic heating, horizontal vorticity, and updrafts, can yield PV anomalies through the latent heating and tilting processes.

The strength of PV anomalies in UWNMS simulations will be shown to be primarily related to the associated mesoscale horizontal wind pattern and resulting relative vorticity maxima. We will use (2) to evaluate the hypothesis that the tilting term is sufficient to account for the time rate of change of PV anomalies in the model. The role of vertical compression 


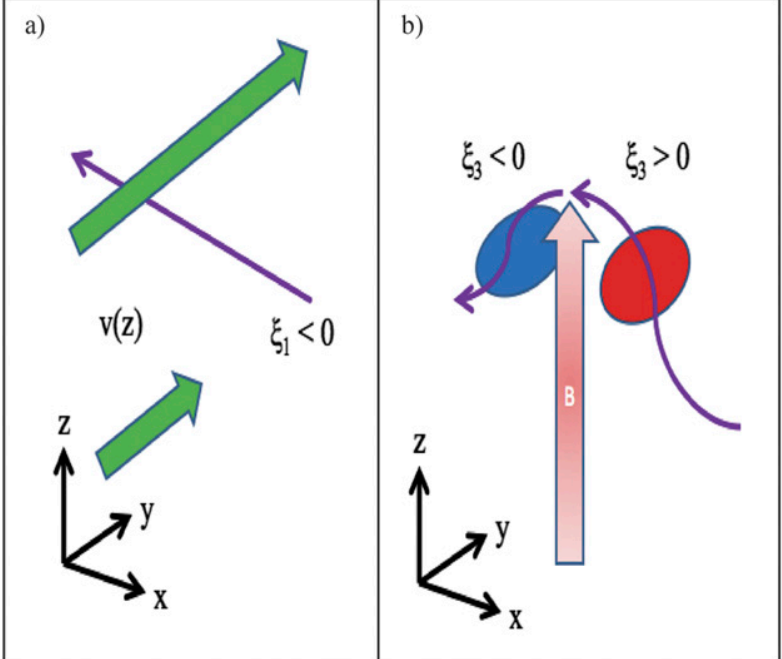

FIG. 2. Mental model for the origin of UTLS PV dipoles, showing (a) an idealized case of northward shear (westward horizontal vorticity sheet or collection of westward-pointing vortex tubes), with (b) a convective updraft that tilts the vortex tube into a horseshoe shape, generating a positive PV anomaly to the right and a negative PV anomaly to the left of the wind shear direction ( $+y$ direction).

and enhancement of static stability at the base of the stratosphere above an updraft will also be discussed with regard to diagnosing the strength of UTLS EPV anomalies seen in simulations.

The tilting term provides an understanding of how a horizontally oriented vortex tube or sheet can be bent by a convective updraft into a vorticity dipole as seen in plan view. Consider all three components of vorticity $\left(\zeta_{1}, \zeta_{2}, \zeta_{3}\right)$, where $\zeta_{1}$ corresponds to vorticity aligned along the $x$ axis, $\zeta_{2}$ along the $y$ axis (e.g., $\Lambda$ ), and $\zeta_{3}$ along the $z$ axis (of primary interest for PV dipoles in the UTLS). Figure 2 is an attempt to combine Chagnon and Gray's Boussinesq study with the phenomenological description of an updraft creating a horseshoe-like deformation of a horizontally oriented vortex tube, thereby producing a horizontal PV dipole.

A strong convective updraft will bend a horizontally oriented vortex tube into the vertical, with a positive PV anomaly to the right and a negative PV anomaly to the left of the direction looking downwind in the UTLS (green arrows in Fig. 2a). This suggests that dipoles of potential vorticity and potential temperature tend to be orthogonal to each other. By analogy with Buys Ballot's law, where, if one stands with one's back to the wind, looking downwind, low pressure lies to the left-a statement of quasigeostrophic flow-we suggest a rule of thumb for convectively generated PV dipoles, where one expects "low PV to the left" of the ambient vertical wind shear. This concept is integral to our diagnosis of convective dipoles in northeastward shear flow in midlatitude cyclones (Fig. 2a). It also proves to be useful in diagnosing PV dipoles arising from convection penetrating upper-tropospheric azimuthal wind shear in tropical cyclones. If one considers the region of a tropical cyclone just to the east of the surface low in the Northern Hemisphere, then upper-tropospheric shear is expected to be southward (cyclonic in the midtroposphere becoming anticyclonic in the upper troposphere). Thus, a convective PV dipole in this location would be expected to comprise a positive anomaly to the west and a negative anomaly to the east (to the left of the southward shear). Considering that the axisymmetric circulation in a tropical cyclone maximizes in the middle troposphere, the rule of thumb may be stated "negative PV lies radially outward."

An interesting aspect of producing PV dipoles in the UTLS is that the anticyclonic member can become inertially unstable: $f\left(\zeta_{3}+f\right)<0$. It will be shown that the outflow from the negative half of the dipole tends to be larger than that from the positive half. Simulations of Tropical Cyclone Ita in the Southern Hemisphere (not shown) are consistent with the general principle that negative PV anomalies are found to the left of the ambient wind shear. However, in the Southern Hemisphere, it is the positive PV anomaly that can become inertially unstable, since positive PV is anomalous PV for the Southern Hemisphere. Generalizing the rule of thumb to include the Southern Hemisphere, "anomalous PV lies radially outward."

In numerical simulations of complex behavior in midlatitude cyclones, an enhanced jet in the UTLS should be expected to occur between PV dipoles. PV dipoles generated by convection must be self-consistent with the horizontal wind field, with an upper-tropospheric mesoscale jet necessarily accompanying a PV dipole. In the case of Talas, the mesoscale jet formed by a long-lasting PV dipole, together with development of high-amplitude quasi-stationary gravity waves, created a quasi-stable dynamical configuration, which became a dominant feature of the tropical cyclone.

\section{Data and analysis methods}

These case studies are modeled with the UWNMS (Tripoli 1992; Pokrandt et al. 1996; Jascourt 1997; Mecikalski and Tripoli 1998), initialized with $2.5^{\circ}$ European Centre for Medium-Range Weather Forecasts (ECMWF) global analyses. The initial UWNMS resolution for both cases was $20 \mathrm{~km} \times 20 \mathrm{~km} \times 300 \mathrm{~m}$, with a grid volume of $152 \times 152 \times 60$ points. The model domain 


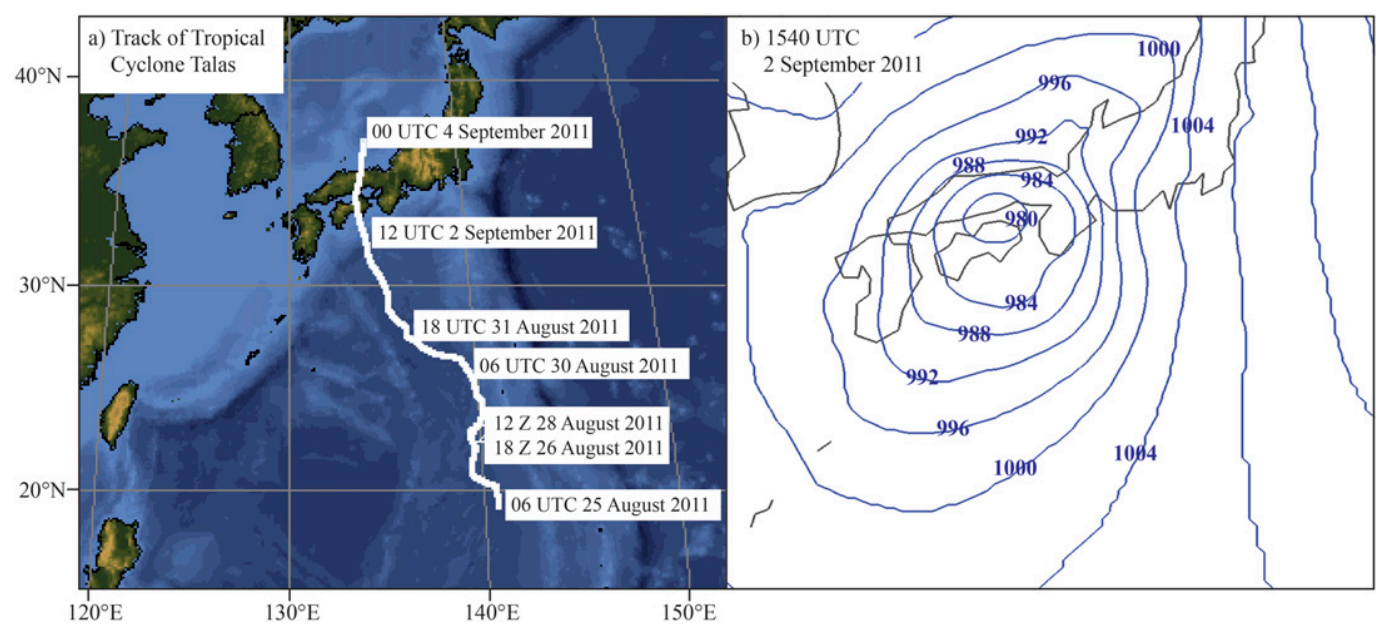

FIG. 3. (a) Track of Tropical Cyclone Talas (white line) as it develops over the western Pacific Ocean near $20^{\circ} \mathrm{N}$, $140^{\circ} \mathrm{E}$ on 25 Aug 2011 and ends in the Sea of Japan near $38^{\circ} \mathrm{N}, 133^{\circ} \mathrm{E}$ on 4 Sep 2011 (image courtesy SSEC). (b) UWNMS simulation of sea level pressure (black contours; $4 \mathrm{hPa}$ ) at 1540 UTC 2 Sep 2011.

for the 20-km-resolution Talas study was chosen to be $31^{\circ}-46^{\circ} \mathrm{N}$ and $125^{\circ}-145^{\circ} \mathrm{E}$, with the center point at $33^{\circ} \mathrm{N}$, $135^{\circ} \mathrm{E}$. The model domain for the Super Tuesday storm spans $26.4^{\circ}-53.6^{\circ} \mathrm{N}$ and $112.8^{\circ}-77.15^{\circ} \mathrm{W}$, with the center point at $40^{\circ} \mathrm{N}, 95^{\circ} \mathrm{W}$. The top of the model was set to $25 \mathrm{~km}$ for Tropical Cyclone Talas and $16 \mathrm{~km}$ for the midlatitude Super Tuesday storm, which is located $\sim 7 \mathrm{~km}$ or $\sim 24$ vertical grid points above the UTLS PV dipole structures of interest in each case.

The dependence of PV dipole structures on model resolution was explored for the Talas case study by comparing simulations at 20-, 15-, and $10-\mathrm{km}$ resolution.

Convective parameterizations were turned off for all simulations. We use Vis5D to illustrate the 3D structure of the PV dipoles and their dynamical environment in the UWNMS. Soundings of winds and PV in the UWNMS were digitized from Vis5D images and replotted in MATLAB.

Selected images were obtained from the Space Science and Engineering Center (SSEC; http://www.ssec. wisc.edu/data/) for the Talas case study. These include cyclone-track maps, visible satellite cloud images with cloud-top winds, and infrared satellite images highlighting cloud tops.

\section{Case study 1: Tropical Cyclone Talas (2-4 September 2011)}

\section{a. Talas synopsis}

Figure $3 \mathrm{a}$ shows the path of Tropical Cyclone Talas as it carved a slow track northward from 25 August 2011 in the north Philippine Sea, over southwestern Japan, ending on 4 September 2011 in the Sea of Japan. We focus on simulating the landfall and transition of Talas during 2-4 September. Talas is notable for bringing record-breaking rainfall to extensive areas of Japan, including the mountains of the Kii Peninsula, where $\sim 1.6 \mathrm{~m}$ of rain fell in a 72-h period (Oku et al. 2014). In some areas of the Kii Peninsula, total rainfall exceeding $2 \mathrm{~m}$ was estimated based on rain gauge-calibrated radars (Japan Meteorological Agency 2011). This heavy rainfall triggered extensive slope failures on the Kii Peninsula (Yamada et al. 2012).

Figure $3 b$ shows sea level pressure in the UWNMS at 1540 UTC 2 September 2011 for a portion of the model centered over southeast Japan. At this time, the central pressure of Talas was $980 \mathrm{hPa}$, with strong onshore winds to the east of the low bringing rain into the Kii Peninsula. During 2-3 September, the northward progression of Talas slowed considerably upon landfall.

Film sequences of visible cloud images with uppertropospheric winds, enhanced infrared images, and upper-tropospheric water vapor images, emphasizing deep convective clouds and cirrus blow-off at the top of the cyclone (http://ssec.wisc.edu/data) were examined to select time periods of unusually robust, long-lasting convection to the east of the surface low. Talas evolved in a complex environment, beginning as the third cyclone in a chain traveling westward south of Japan, and succeeded by a fourth cyclone landfalling near Tokyo from the east a few days later. As Talas approached landfall during 2 September, a series of three strong convective centers, located $\sim 100 \mathrm{~km}$ east of Talas' surface low, slowly propagated northward into the Kii Peninsula. The third convective event was particularly 


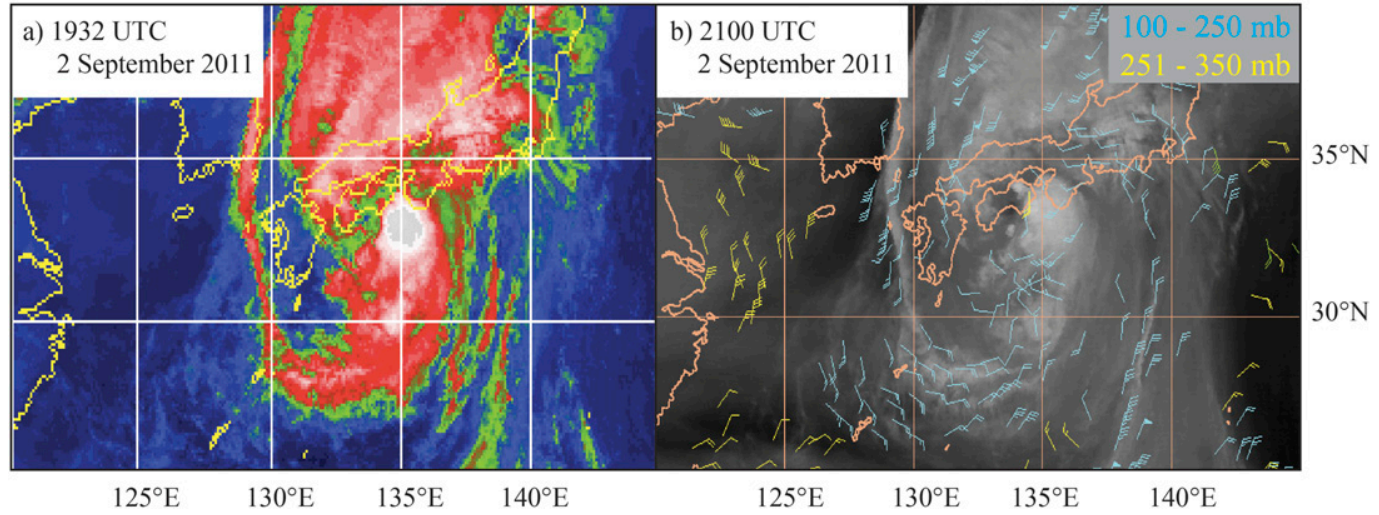

FIG. 4. (a) Infrared satellite image of Tropical Cyclone Talas during a strong convective outbreak to the east of the low at 1932 UTC 2 Sep 2011, with increasingly cold and high clouds from blue to green to red to white. (b) Upper-tropospheric water vapor satellite image at 2100 UTC 2 Sep2011, with wind barbs (blue for 100-250-hPalayer winds and yellow for 251-350-hPa-layer winds). Images courtesy of SSEC.

strong and persisted from $\sim 1400$ UTC 2 September through $\sim 0200$ UTC 3 September, about $12 \mathrm{~h}$ in duration. After this time the central low passed northward over land, with no further occurrence of robust convective events, and Talas rapidly dissipated as it traveled northward over the Sea of Japan.

Figure 4 shows a satellite image in the infrared at 1932 UTC 2 September, together with upper-tropospheric water vapor and winds at 2100 UTC 2 September, during the height of the third convective event. Note the deep convective region just to the south of the Kii Peninsula, with cirrus spiraling anticyclonically outward from Talas in the UTLS. The convective complex of interest is located at $\sim 33^{\circ} \mathrm{N}, 135^{\circ} \mathrm{E}$, about $100 \mathrm{~km}$ to the east of the surface low.

\section{b. Convective towers and PV dipoles in Talas}

The horizontal structure of $\theta_{e}$, EPV, divergence, and winds at $16.5-\mathrm{km}$ altitude is shown in Fig. 5 during the development (1720 UTC 2 September) of the prominent convection seen near $33^{\circ} \mathrm{N}, 135^{\circ} \mathrm{E}$ (Fig. 4). At this level, a modified theta dipole is seen (Fig. 5a), with a cold dome (blue) centered on the updraft (white), with a ring of warmer values, enhanced to the east and south of the updraft. This may be viewed as consistent with Fig. 1 if one assumes an idealized anticyclonic circulation at the top of a tropical cyclone.

Figure $5 \mathrm{~b}$ shows that the PV dipole at $16.5 \mathrm{~km}$ straddles the updraft and is displaced toward the SSE from the updraft. The PV dipole anomalies exceed \pm 30 PVU, implying that the negative center is inertially unstable. At this location in the UWNMS, $f \sim 2 \times 10^{-5} \mathrm{~s}^{-1}$, $\rho \sim 0.1 \mathrm{~kg} \mathrm{~m}^{-3}$, and $\partial \theta / \partial z \sim 30 \mathrm{~K} \mathrm{~km}^{-1}$. An estimate of PV without relative vorticity $\left(\zeta_{3} \sim 0\right)$ is $\sim 6$ PVU. Therefore, mesoscale horizontal wind shears on the order of $\pm 5 f \sim 1 \times 10^{-4} \mathrm{~s}^{-1}$ are required to achieve PV anomalies of \pm 30 PVU.

Figure $5 \mathrm{c}$ shows the region of divergence associated with the convective updraft, exceeding $-60 \times 10^{-5} \mathrm{~s}^{-1}$ near the center of the updraft. Another divergence maximum occurs to its west, associated with a second updraft. Figure 5d shows the horizontal circulation pattern associated with the PV dipole and the updraft. A distinctive cyclonic circulation is evident around the positive PV quadrant (red shading), and an anticyclonic circulation can be seen around the negative PV quadrant. Outflow from the updraft circulates preferentially poleward and eastward around the negative PV anomaly. There is a significant asymmetry in the strength of the circulation, with that around the positive PV anomaly being weaker. We hypothesize that the inertial instability of the negative anomaly provides a more favorable environment for outflow from the updraft, with correspondingly higher wind speeds in the anticyclonic outflow, as the pressure gradient force from the center of the updraft accelerates mesoscale flow toward geostrophy.

At this altitude, horizontal winds associated with the PV dipole dominate, as the large-scale flow around Talas diminishes upward into the lower stratosphere. The PV anomalies exceed $\sim 300 \mathrm{~km}$ in meridional extent and $\sim 200 \mathrm{~km}$ in zonal extent. Assuming that horizontal winds near the anomalies range from $\sim 0 \mathrm{~m} \mathrm{~s}^{-1}$ in the center to $\sim 20 \mathrm{~m} \mathrm{~s}^{-1}$ at $100-\mathrm{km}$ radial distance, then $|\partial v / \partial x| \sim 2 \times 10^{-4} \mathrm{~s}^{-1}$, which is the right order of magnitude to account for the \pm 30 -PVU anomalies.

What would be required for PV anomalies of order \pm 30 PVU to be produced in $1 \mathrm{~h}$ ? From (2), $(D / D t) \zeta_{3} \sim-[(\partial w / \partial x)(\partial v / \partial z)]$, this would require the tilting term to produce relative vorticity anomalies at the 


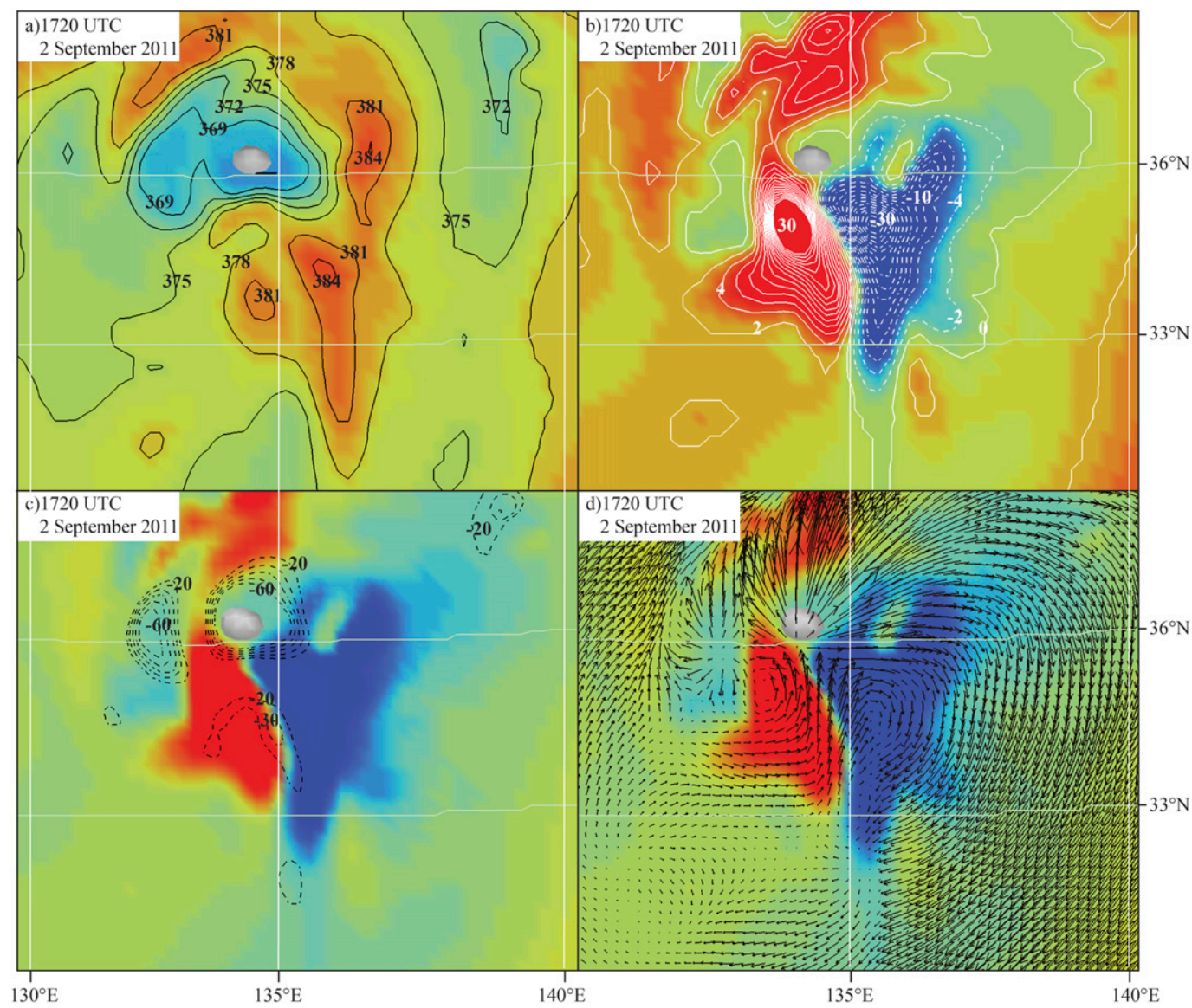

FIG. 5. Plan views at $16.5 \mathrm{~km}$ at 1720 UTC 2 Sep 2011 near the updraft centered at $33^{\circ} \mathrm{N}, 135^{\circ} \mathrm{E}$ (white isosurface of $50 \mathrm{~cm} \mathrm{~s}^{-1}$ upward motion) in the UWNMS simulation of Talas of (a) $\theta_{e}$ (black contours and color, with blue $<372 \mathrm{~K}$ and red $>390 \mathrm{~K}$ ); (b) EPV (PVU; white contours and shading; red is strongly positive and blue is negative); (c) as in (b), but with divergence (black dashed contours with interval of $1 \times 10^{-5} \mathrm{~s}^{-1}$ from $1 \times 10^{-5}$ to $10 \times 10^{-5} \mathrm{~s}^{-1}$ ); and (d) as in (b), but with horizontal wind vectors (largest vector is $40 \mathrm{~m} \mathrm{~s}^{-1}$ ).

rate $\sim\left(1 \times 10^{-4} \mathrm{~s}^{-1}\right) / 3600 \mathrm{~s} \sim 3 \times 10^{-8} \mathrm{~s}^{-2}$. Taking $w \sim$ $1 \mathrm{~m} \mathrm{~s}^{-1}, \delta x \sim 100 \mathrm{~km}, \delta v \sim 50 \mathrm{~m} \mathrm{~s}^{-1}$, and $\delta z \sim 10 \mathrm{~km}$, the tilting term can cause $\left|(D / D t) \zeta_{3}\right| \sim 5 \times 10^{-8} \mathrm{~s}^{-2}$. This supports the mechanism of updraft tilting of ambient horizontal vorticity.

Figure 6 shows vertical soundings from the UWNMS in the layer through the positive (Figs. 6a,b) and negative (Figs. 6c,d) PV anomalies in Fig. 5. The vertical extent of each PV anomaly is $\sim 3 \mathrm{~km}$ (10 grid points). The most positive PV region of the dipole (black profile in Figs. 6a and 6b) exceeds 70 PVU in the 16-19-km layer, while the most negative EPV region of the dipole (black profile in Figs. 6c and 6d) is nearly $-60 \mathrm{PVU}$ in this layer.

The ambient wind shear in the upper troposphere above the poleward cyclonic wind maximum $(\sim 6 \mathrm{~km})$ is $\partial v / \partial z \sim-50 \mathrm{~m} \mathrm{~s}^{-1}$ in the layer from 6 to $16 \mathrm{~km}$ (blue profile in Fig. 6a), confirming the magnitude used to estimate the tilting term above. In this case, the positive PV anomaly is located closer to the updraft (cf. Fig. 5), so vertical motion is more pronounced (cf. Figs. 6a,c). The structure above $16 \mathrm{~km}$ is complicated by the existence of the PV dipole, where the local circulation includes a northward jet between the dipole (peak in meridional flow near $17 \mathrm{~km}$ in Figs. 6a and 6c).

The PV anomalies lie above $16 \mathrm{~km}$. In the layer $16-$ $18 \mathrm{~km}$, potential temperature increases upward into the stratosphere at a rate of $30 \mathrm{~K} \mathrm{~km}^{-1}$ in the UWNMS. This implies that static stability is strongly positive in both the negative and positive PV anomalies. The enhanced static stability in this layer helps to amplify the absolute vorticity anomalies when converted to PV.

The right-hand panels of Fig. 6 show a comparison among profiles of EPV (black), absolute vorticity (brown), and absolute vorticity scaled by density (gray). Values of EPV are in PVU. Values of absolute vorticity 

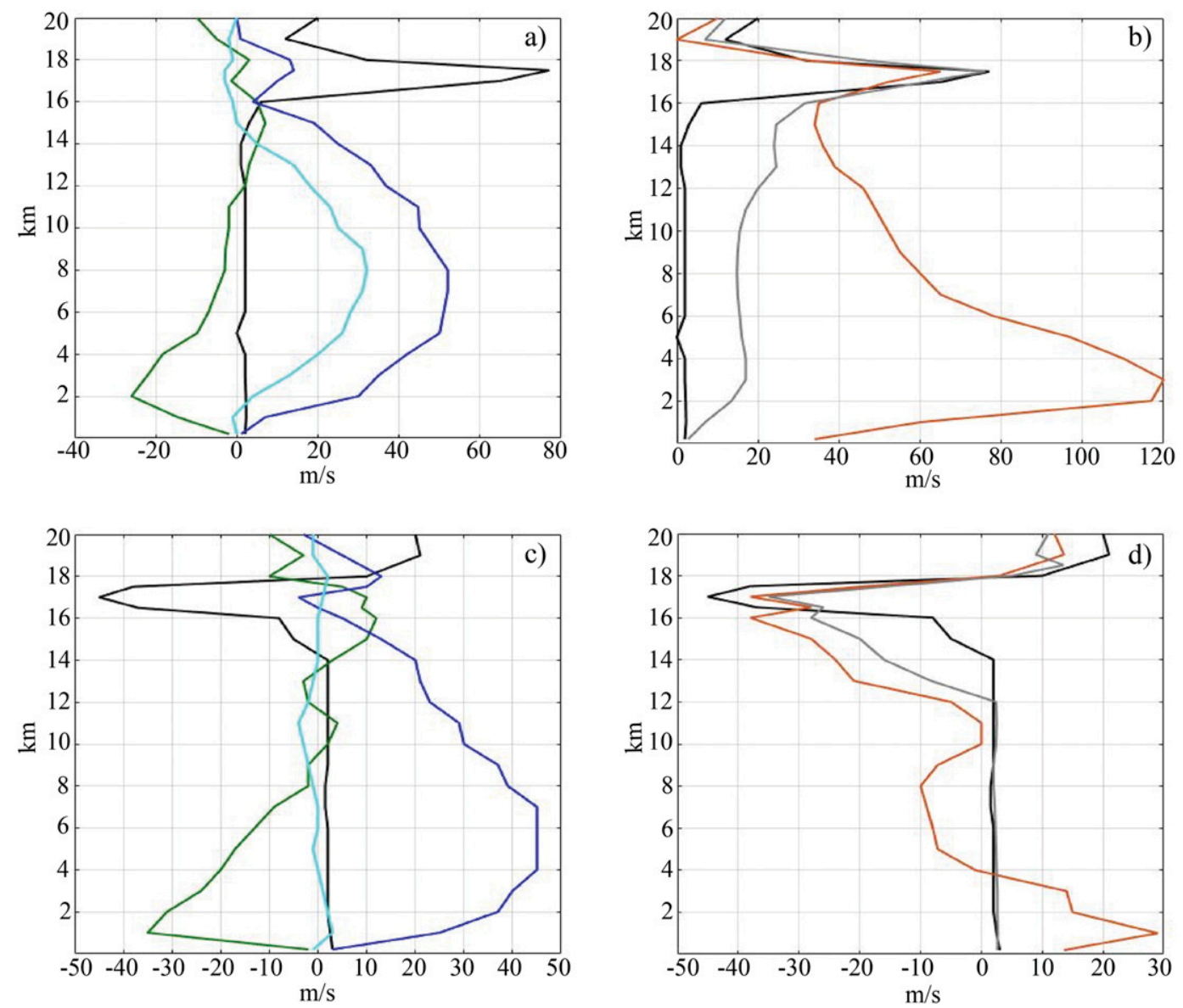

FIG. 6. Vertical sounding from 0- to 25-km altitude in the UWNMS simulation of Talas, sampled in the center of the (a),(b) positive and (c),(d) negative members of the PV dipole at 1540 UTC 2 Sep 2011 shown in Fig. 5. (a),(c) Profiles of EPV (black; PVU), zonal wind (green; $\mathrm{m} \mathrm{s}^{-1}$ ), meridional wind (dark blue; $\mathrm{m} \mathrm{s}^{-1}$ ), and vertical wind (light blue; $\mathrm{m} \mathrm{s}^{-1} \times 10$ ). (b),(d) Profiles of EPV (black; PVU), absolute vorticity (brown; $\mathrm{s}^{-1} \times 10^{5}$ ), and absolute vorticity scaled by density (silver; PVU; see text).

are in units of $\mathrm{s}^{-1} \times 10^{5}$. Profiles of absolute vorticity were scaled by density using $1 / \rho \sim\left(p_{o} / \rho_{o}\right)(1 / p)$, where $p$ is model pressure, and the subscript 0 indicates surface values. This variable is also divided by 10 to convert to PVU for comparison to PV near 16.5-km altitude, where $1 / \rho \sim 10 \mathrm{~m}^{3} \mathrm{~kg}^{-1}$ and $\partial \theta / \partial z \sim 0.01 \mathrm{~K} \mathrm{~m}^{-1}$. Note that peak $\mathrm{PV}$ values of $\pm 50 \mathrm{PVU}$ correspond to absolute vorticity values of $\pm 5 \times 10^{-4} \mathrm{~s}^{-1}$. A comparison of EPV and PV is not shown because the profiles are nearly indistinguishable. In the convective updraft both moist and static instability are near zero, so both EPV and PV are near zero in the updraft, while in the lower stratosphere they differ negligibly because it is so dry.

There is good agreement between the magnitudes of EPV, absolute vorticity, and density-scaled absolute vorticity in both PV anomalies (Figs. 6b,d). Below $16 \mathrm{~km}, \mathrm{EPV}$ and PV are near zero. In the convective updraft below the positive PV maximum (Fig. 6b), cyclonic absolute vorticity is found throughout the column, while density-scaled absolute vorticity exhibits a downward diminution in magnitude. Below the negative PV maximum (Fig. 6d) cyclonic absolute vorticity becomes anticyclonic in the midtroposphere, with an upward increase into the negative PV maximum above $10-\mathrm{km}$ altitude. It will be shown that this difference in the vertical profiles of absolute vorticity below the positive and negative maxima is also found in the midlatitude convective case. Again, scaling by density diminishes values at lower altitudes relative to absolute vorticity.

Consider the ideas in Fig. 2 for the flow geometry located to the east of the center of Talas, where northward flow diminishes from $\sim 50 \mathrm{~m} \mathrm{~s}^{-1}$ at $6 \mathrm{~km}$ to $\sim 0 \mathrm{~m} \mathrm{~s}^{-1}$ at $16 \mathrm{~km}$ (Figs. 6a,c). Southward vertical shear is diagnosed to the east of the center of Talas in the upper troposphere, with associated horizontal vorticity pointed radially away from the center of Talas (directed eastward 
at this location), so that $\zeta_{1}>0$. In this configuration an updraft would be expected to tilt this horizontal vorticity upward to the west of the updraft $\left(\zeta_{3}>0\right)$ and downward to the east of the updraft $\left(\zeta_{3}<0\right)$. Since uppertropospheric shear is directed southward, the PV dipole in Fig. 5 is consistent with the suggested rule of thumb that the low-PV anomaly lies to the left of the wind shear, or radially outward.

Supplementary Fig. S1 shows a movie of trajectories that pass through the positive and the negative UTLS PV anomalies. It illustrates that the PV anomalies are distinctly associated with parcels ascending from the boundary layer in the convective updraft and entering the base of the stratosphere.

The vertical structure of winds and PV anomalies at 1540 UTC 2 September in the 16-18-km layer are shown in Fig. 7. The divergent outflow from the updraft is most notable at $16 \mathrm{~km}$, with outflow to the north and east curving anticyclonically southward to the southeast of Japan. At $16 \mathrm{~km}$, the PV anomalies are $\sim \pm 10$ PVU but increase to \pm 30 PVU at $17 \mathrm{~km}$ and then diminish slightly at $18 \mathrm{~km}$. At increasing altitude the horizontal flow diminishes upward from maximum values of $50 \mathrm{~m} \mathrm{~s}^{-1}$ at $16 \mathrm{~km}$ to $35 \mathrm{~m} \mathrm{~s}^{-1}$ at $17 \mathrm{~km}$ and $15 \mathrm{~m} \mathrm{~s}^{-1}$ at $18 \mathrm{~km}$. At $18 \mathrm{~km}$, the PV structure is more complex, but a persistent, northward mesoscale jet over Japan is still noticeable.

The presence of a significant mesoscale northward jet between the PV dipole (Figs. 5d, 7) strongly resembles the idealized PV dipole jet studies described in the introduction. This jet is sufficient to overwhelm the modest southward flow expected in symmetric anticyclonic outflow from Talas, reversing the upper-tropospheric southward vertical wind shear to northward in the 1618-km layer. When the PV dipole becomes highly amplified, the normal vertical shear in a tropical cyclone can be reversed. Continuous northward flow may be found to the east of the surface low extending from the surface to $18 \mathrm{~km}$ in this event. The basic-state shear may be termed "linear" in the sense of the basic-state, symmetric circulations expected around tropical cyclones, but the coupled system with a strong PV dipole is "nonlinear" in the sense that the PV dipole determines local shear in the UTLS. Excitation of a strong PV dipole coincides with the formation of a strong field of gravity waves, which amplifies the updrafts and downdrafts (not shown).

\section{c. Extratropical transition of Talas: From PV dipoles to PV streamers}

Numerous studies have examined tropical cyclones as they undergo transition into the midlatitudes in terms of energy budgets and structural changes (Palmen 1958;
DiMego and Bosart 1982; Klein et al. 2000; Kitabatake et al. 2007; Kitabatake 2008). Agusti-Panareda et al. (2004) used an idealized simulation of Hurricane Irene and tracked the PV features into the extratropics using a PV inversion process. Extratropical transition associated with most tropical cyclones is a complex process, unique to each individual cyclone, as the position of the subpolar and subtropical jet and the associated troughs and ridges differ from storm to storm. Here the evolution of PV structures will be employed as a descriptor of the extratropical transition of Tropical Cyclone Talas.

Figures 8 and 9 show the 15 -h evolution of EPV and streamfunction at $17 \mathrm{~km}$ for the region of convective PV dipoles, as Talas underwent an extratropical transition from 1420 UTC 2 to 0520 UTC 3 September. The initial formation of the primary EPV dipole in Fig. 5 may be seen centered near $34^{\circ} \mathrm{N}$ in Fig. $8 \mathrm{a}$ (1420 UTC). It is characterized by a somewhat irregular structure and a large spatial extent, spanning $3^{\circ}$ of latitude, with PV anomalies of $\sim \pm 20$ PVU. Blue regions in Fig. 8 highlight negative PV, or inertially unstable air. By 1800 UTC (Fig. 8b), the structure became more complex and widespread, with a noticeable increase in the area of the negative PV anomaly. By 1920 UTC (Fig. 8c) the PV structures began to elongate meridionally. The positive PV anomaly now spans over $7^{\circ}$ of latitude (cf. Fig. 9c), but the negative anomaly is still fairly symmetric. By 2140 UTC (Fig. 8d) the negative PV anomaly has broken into a series of meridionally elongating PV streamers, related to gravity wave formation. As Talas progressed northward into the Sea of Japan at 0240 (Fig. 8e) and 0520 UTC 3 September 2011 (Fig. 8f), the original PV anomalies became more and more elongated. A negative PV streamer may be seen extending from south of $33^{\circ} \mathrm{N}$ to poleward of $42^{\circ} \mathrm{N}$. This structure is spatially similar to the low-PV streamer found on the equatorward side of the subpolar jet downstream of deep convection in the Super Tuesday midlatitude cyclone (cf. Rowe and Hitchman 2015, Fig. 6 and section 5).

The synoptic evolution of the environment of the UTLS PV dipoles spawned by Talas can be gleaned from the evolution of horizontal streamfunction at $17 \mathrm{~km}$, which is shown along with EPV contours in Fig. 9. In the subtropical lower stratosphere, the transition from the prevailing zonal-mean summertime easterlies to a period of westerly flow can occur when a midlatitude trough travels eastward through a region. In the presence of a tropical cyclone with anticyclonic mass outflow, the sum of these processes yielded the situation in Fig. 9a. Prevailing subtropical stratospheric easterlies are deflected southward around the anticyclonic outflow from Talas, then join with the northeastward flow in advance of a synoptic trough. The 


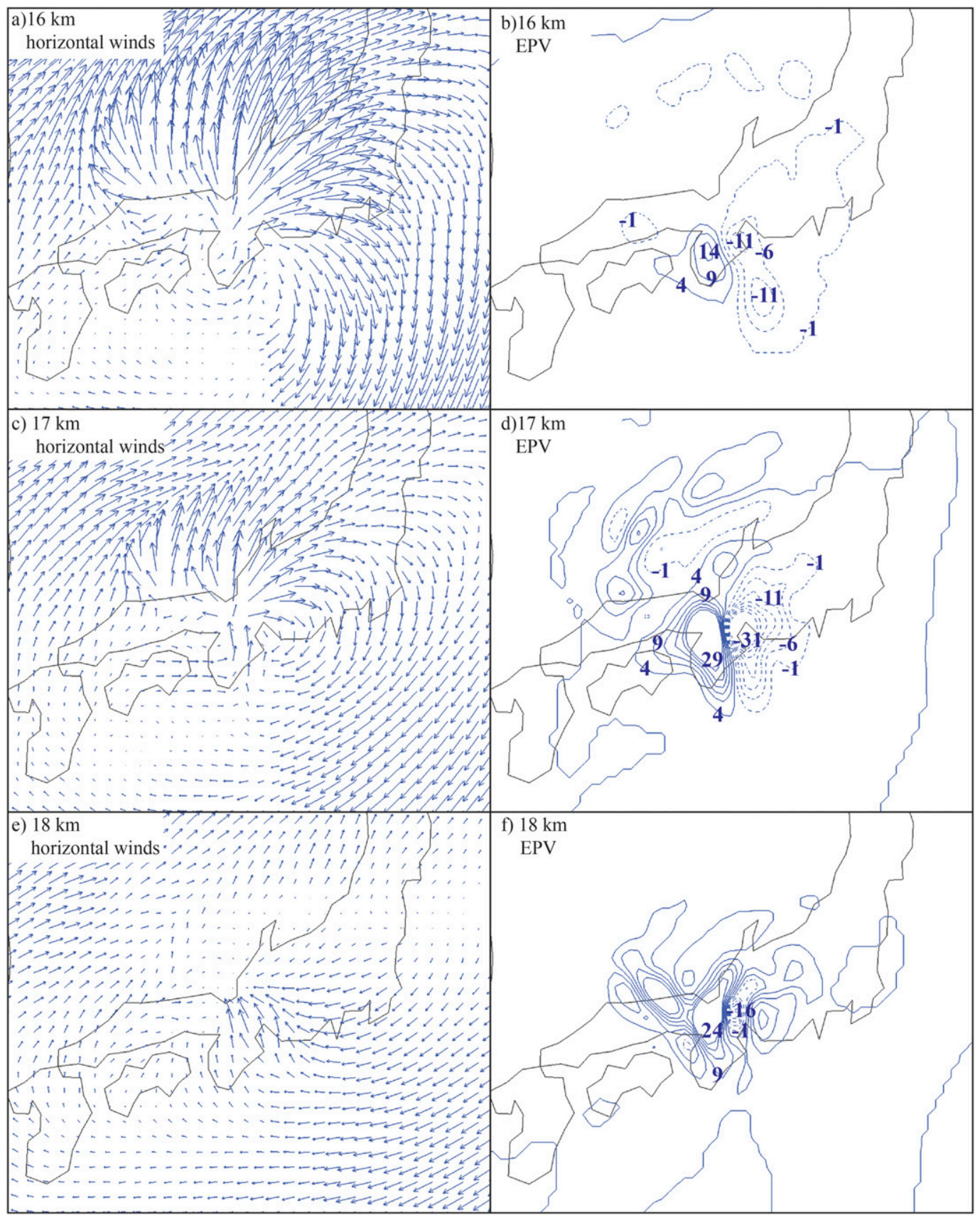

FIG. 7. UWNMS simulation of Talas at 1540 UTC 2 Sep 2011 showing the spatial relationship between wind shear and circulation patterns associated with the horizontal EPV dipole formation at (a),(b) 16, (c),(d) 17, and (e),(f) $18 \mathrm{~km}$. (left) Wind vectors (blue arrows) and (right) horizontal EPV anomalies (blue contours; dashed is negative; solid is positive). The longest vectors are (a) 50 , (b) 35 , and (c) $15 \mathrm{~m} \mathrm{~s}^{-1}$.

deformation associated with the wind pattern in Fig. 9a would be expected to act on an idealized symmetric PV anomaly by elongating it poleward on its western side and equatorward on its eastern side. The fundamental anticyclonic quality of the flow near Talas reflects the regional effects of deep convection, while the hairpin shape of the flow is produced by the large-scale deformation field.

From 1440 to 2140 UTC (Figs. 9a-d) the poleward flow between the PV dipole grows in strength as the upward-growing speed maximum (Figs. 6, 7) manifests itself in the lower stratosphere. The persistence of this 


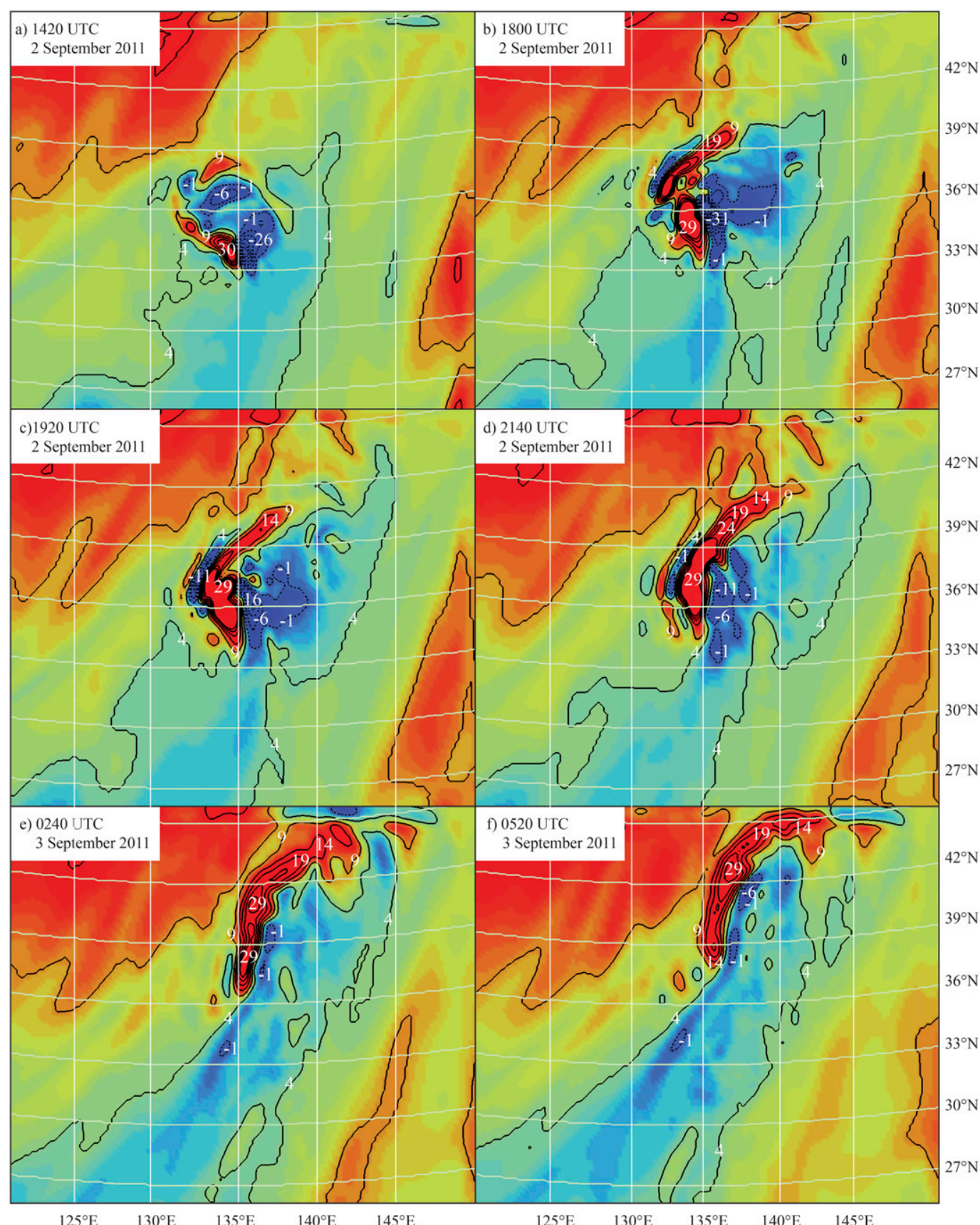

FIG. 8. Time sequence of $17-\mathrm{km}$ EPV in the UWNMS associated with the extratropical transition of Talas at (a) 1420, (b) 1800, (c) 1920, and (d) 2140 UTC2 Sep and (e) 0240 and (f) 0520 UTC 3 Sep 2011. EPV is shaded (red is positive; blue is negative) with labels at color transitions every 3 PVU starting at 1 PVU.

mesoscale dipole jet maximum is similar to the results of Deng et al. (2012). Upon its passing over land, the convection began to diminish. As the trough slowly propagated in from the west and Talas propagated northward, the PV dipoles at $17 \mathrm{~km}$ entered strong northward flow in persistent strong horizontal shear. The evolution of EPV, vertical motion, and horizontal winds in the UTLS during this extratropical transition may be viewed in supplemental movies S2 and S3.

This pair of PV streamers, with an elongated positive streamer just to the west and poleward of an elongated negative streamer, is consistent with a strengthening subtropical jet in the UTLS. Rowe and Hitchman (2016) showed that the existence of an inertially unstable 

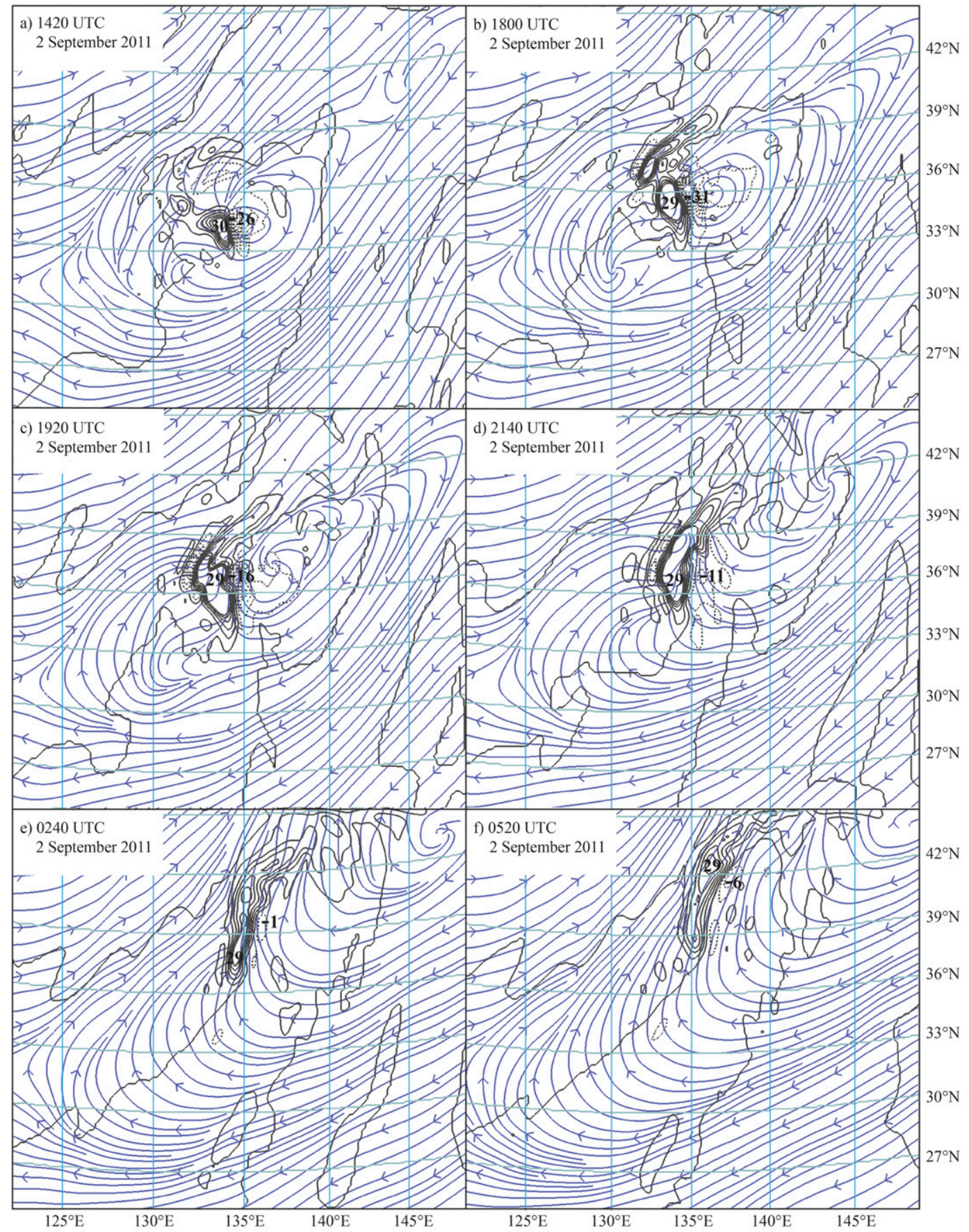

FIG. 9. As in Fig. 8, but showing the relationship between 17-km streamfunction (blue) and EPV (black contours at interval of $4 \mathrm{PVU}$, with zero contour deleted for clarity).

negative PV streamer can help shape the westerly jet by leading to enhanced poleward excursions of parcel trajectories, with the resulting zonal wind maximum displaced toward a higher latitude.

This pair of PV streamers is oriented in the opposite sense to the "low PV to the left" rule, under the assumption that the original convective PV dipoles were created in upward diminishing northward flow (southward shear). But in the presence of the synoptic-scale deformation field, as the convection started to diminish in strength, both the positive anomalies to the west and the negative anomalies to the east were sheared out 


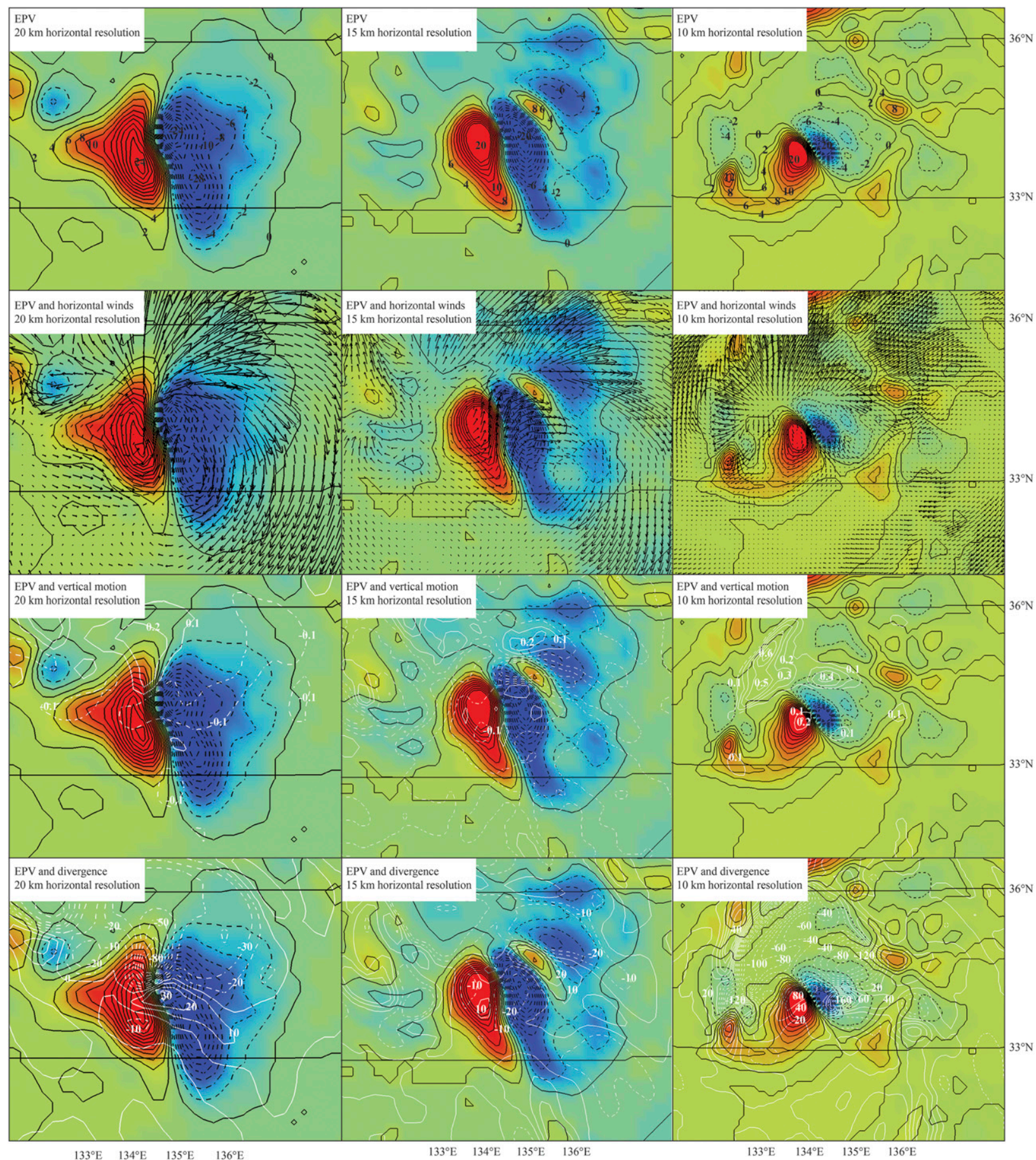

FIG. 10. UWNMS simulation of Tropical Cyclone Talas at 1500 UTC 2 Sep 2011 comparing patterns of 16.5-km EPV at horizontal resolutions of (left) 20, (center) 15, and (right) $10 \mathrm{~km}$. EPV is in color (blue is negative; red is positive), with black contours at 2-PVU interval (negative dashed). Also shown are (second row) horizontal winds at $16.5 \mathrm{~km}$, with maximum wind vector $40 \mathrm{~m} \mathrm{~s}^{-1}$; (third row) vertical motion (white contours; interval of $0.1 \mathrm{~m} \mathrm{~s}^{-1}$; dashed negative); and (bottom) horizontal convergence (white contours; $10 \times 10^{-5} \mathrm{~s}^{-1}$ contour interval; dashed is divergent flow). 


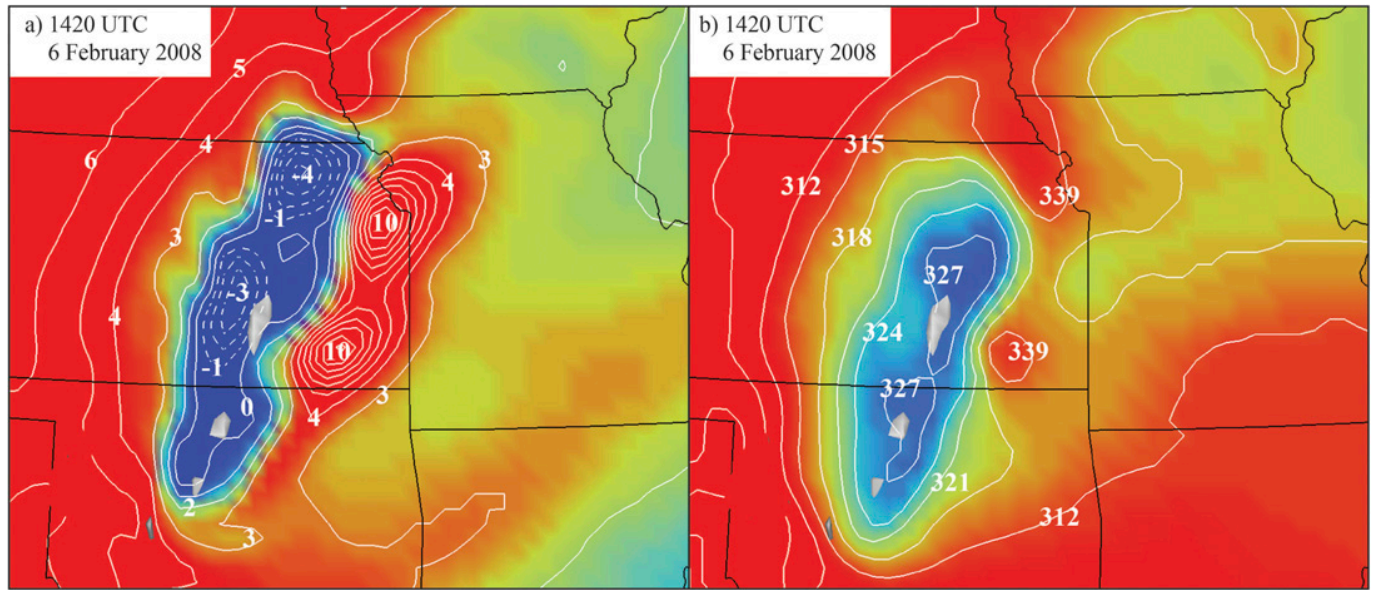

FIG. 11. EPV and theta dipole structures at $11 \mathrm{~km}$ at 1420 UTC 6 Feb 2008 in the UWNMS associated with a strong convective complex, which developed along the cold front in the Super Tuesday storm. (a) Equivalent potential temperature is shown in color, with white contours at an interval of $3 \mathrm{~K}$. The isosurface of vertical motion exceeding $0.6 \mathrm{~m} \mathrm{~s}^{-1}$ is shown in gray. (b) EPV is shown in color (blue is negative; red is positive) with white contours at an interval of 1 PVU (negative dashed).

$(\partial v / \partial x<0)$, elongated meridionally, and advected into the midlatitude westerly jet, altering the jet's structure.

\section{d. Dependence of PV dipole structure on model resolution}

Determination of the dependence of convective structures on model resolution remains a fundamental problem in the atmospheric sciences (Boer and Lazare 1988, Mahlman and Umscheid 1987, Randall et al. 2003, Yamada et al. 2005). As resolution becomes increasingly finer, it is plausible to expect models to resolve individual convective updrafts (Hamilton and Ohfuchi 2008). It is clear that increased resolution generally improves the representation of convectively excited gravity waves important to the adjustment process (Lindzen and Fox-Rabinovitz 1989; Stephan et al. 2016). Here we compare PV structures near convection in the UTLS for horizontal resolutions of 20,15 , and $10 \mathrm{~km}$, keeping all other parameters identical.

Figure 10 shows EPV at $16.5-\mathrm{km}$ altitude for UWNMS resolutions of 20 (left), 15 (center), and $10 \mathrm{~km}$ (right) at 1500 UTC 2 September 2011. The orientation of the PV anomalies, with the positive anomaly closer to the center of the cyclone and the negative anomaly radially farther out, is preserved across this range of model resolution. With increasing resolution, the PV anomalies become somewhat smaller in scale, with more detail at smaller scales.

In the second row of Fig. 10, horizontal wind vectors are superimposed, with maximum wind speeds of $\sim 40 \mathrm{~m} \mathrm{~s}^{-1}$ in each panel. The northward jet between the dipole is preserved across different resolutions, but the predominance of outward flow around the negative anomaly at $20 \mathrm{~km}$ (left) is less pronounced at $10 \mathrm{~km}$ (middle). The third row shows vertical motion contours (white) superimposed on EPV. At increasing resolution more detailed structures in the vertical motion field are found. The bottom row of Fig. 10 shows horizontal divergence superimposed on EPV. Maximum values tend to be near $20 \times 10^{-5} \mathrm{~s}^{-1}$, but with a slight decrease with increasing resolution.

\section{Midlatitude Super Tuesday storm}

The dynamics associated with this destructive midlatitude weather system feature an interesting role for inertial instability in enhancing overfolds in the UTLS and jet intensification (Rowe and Hitchman 2015, 2016). Here we focus on the PV dipole signature of the convection that broke out along the cold front between the subtropical and subpolar jets. Figure 11 shows the UTLS dipoles in EPV and $\theta_{e}$ seen over eastern Nebraska and western Missouri at 1420 UTC 8 February 2008. The linear alignment of several updrafts along the cold front is evident.

An elongated negative $\theta_{e}$ anomaly is seen centered on the chain of updrafts, spanning $5^{\circ}$ latitude, with positive anomalies located to its east and northeast (Fig. 11b). Since the prevailing wind in the UTLS is from the southeast, this cold-warm dipole aligned in the direction of the UTLS wind is consistent with Fig. 1. An elongated EPV dipole may be diagnosed as the result of this linear arrangement of updrafts, with low PV to the left of the wind shear (Fig. 11a), consistent with Fig. 2. In this 


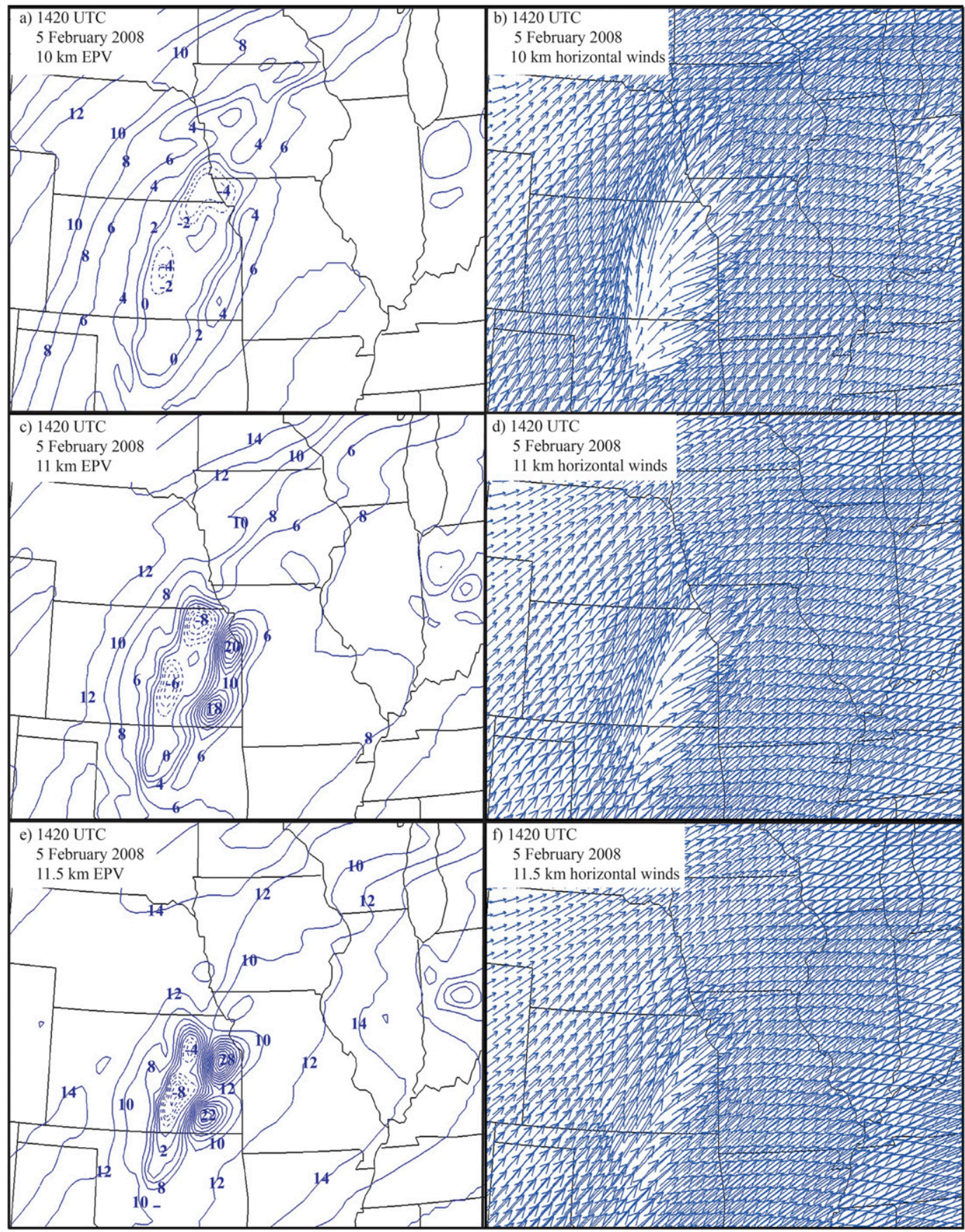

FIG. 12. Structure of (left) EPV (contour interval of 1 PVU; negative contours dashed) and (right) horizontal winds at (a),(b) 10, (c),(d) 11, and (e),(f) $11.5 \mathrm{~km}$ for the UWNMS Super Tuesday simulation. The maximum wind vector is $60 \mathrm{~m} \mathrm{~s}^{-1}$ in (b) and (d) and $50 \mathrm{~m} \mathrm{~s}^{-1}$ in (f).

situation, the cold region is moderately congruent with the negative PV region, which is somewhat different from the convective cluster in Talas. However, the principle that the axes of the theta dipole and PV dipole are perpendicular to each other holds true in both situations.
The patterns of EPV and $\theta_{e}$ seen in Fig. 11 are consistent with the principles evoked in Figs. 1 and 2. Air flowing up and over a chain of updrafts is likely to generate a warm anomaly just downstream. When strong updrafts penetrate the upper-tropospheric northeastward wind shear (northwestward-oriented 

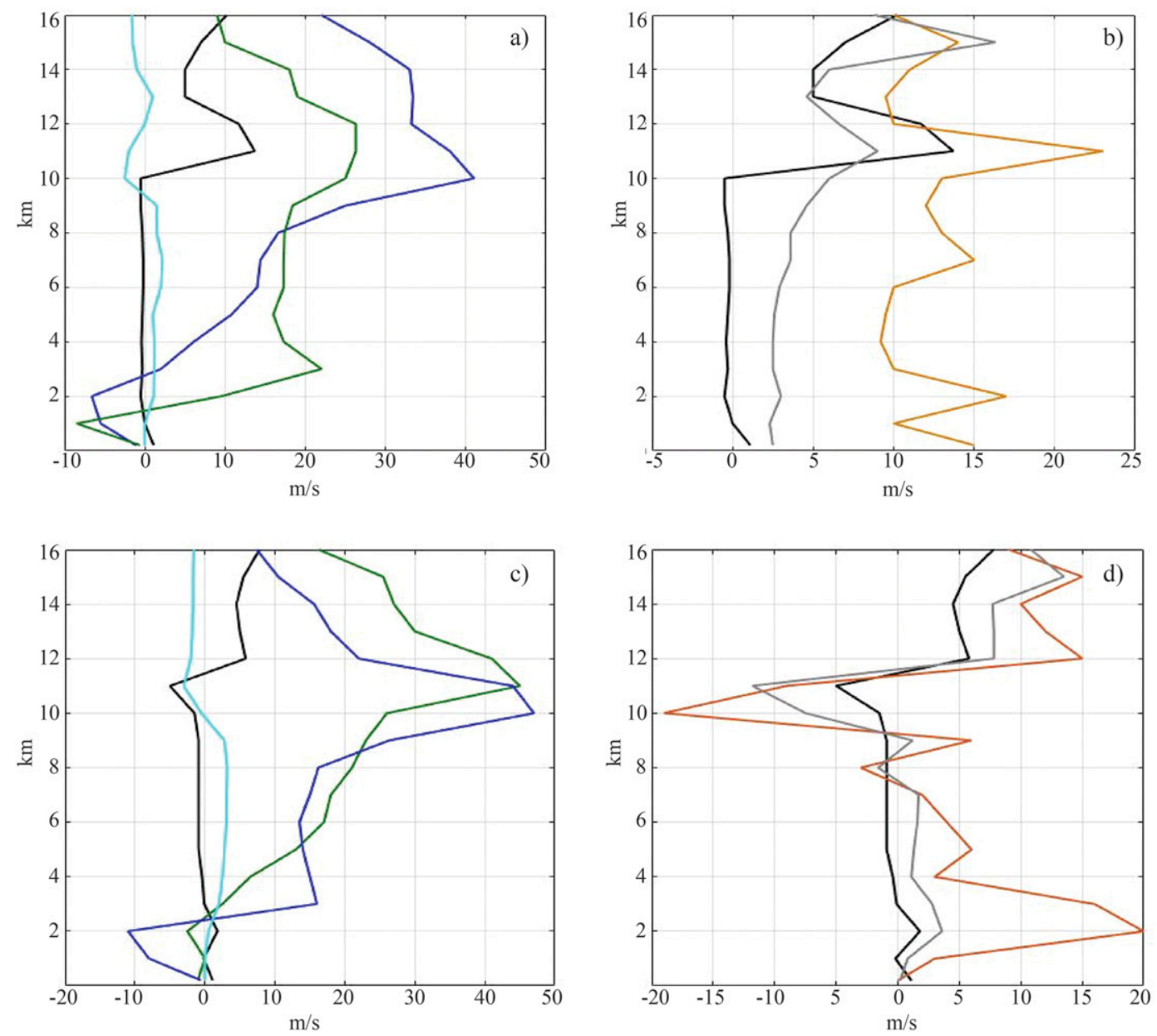

FIG. 13. As in Fig. 6, but for vertical soundings in the center of the (a),(b) positive and (c),(d) negative PV anomalies seen during the Super Tuesday storm in Fig. 11b at 1420 UTC 6 Feb 2008. (a),(c) Profiles of EPV (black; PVU), zonal wind (green; $\mathrm{m} \mathrm{s}^{-1}$ ), meridional wind (dark blue; $\mathrm{m} \mathrm{s}^{-1}$ ), and vertical wind (light blue; $\mathrm{m} \mathrm{s}^{-1} \times 10$ ). (b), (d) Profiles of EPV (black; PVU), absolute vorticity (brown; $\mathrm{s}^{-1} \times 10^{5}$ ), and absolute vorticity scaled by density (silver; PVU; see text).

horizontal vorticity), vorticity will be directed upward (positive, red centers) to the right of the wind shear and directed downward (negative, blue centers) to the left of the wind shear. The individual dipoles associated with each updraft blend into an elongated dipole with low PV to the left of the UTLS wind shear (Fig. 11).

The vertical structure of horizontal winds and EPV in the $10-12-\mathrm{km}$ layer at 1420 UTC 5 February 2008 is shown in Fig. 12. This layer lies immediately above the regional tropopause, analogous to the $16-18-\mathrm{km}$ layer in Fig. 7. At $10 \mathrm{~km}$ (Fig. 12a), the elongated PV dipole can be seen over eastern Kansas, with PV anomalies of order \pm 2 PVU. Note how the prevailing north-northeastward flow is diverted around the divergent outflow from the chain of updrafts (Fig. 12b). At 11-km altitude, values in the PV dipole range from -4 to +10 PVU (Fig. 12c), and the effect of the divergent outflow from the chain of thunderstorms along the cold front is diminishing (Fig. 12d). At $11.5 \mathrm{~km}$, EPV values range from -4 to +14 PVU (Fig. 12e), but the effects of the updrafts on the prevailing horizontal flow is further diminished (Fig. 12f).

Figure 13 shows vertical soundings from the UWNMS sampled in the center of the negative and positive PV anomalies seen in Fig. 11b at 1420 UTC 5 February 2008. EPV anomalies reach -6 and +10 PVU. The northeastward wind shear in the $0-10-\mathrm{km}$ layer is $\sim 50 \mathrm{~m} \mathrm{~s}^{-1}$ $(10 \mathrm{~km})^{-1}$. An evaluation of the relative vorticity associated with the horizontal wind fields seen in Fig. 12 suggests that relative vorticity anomalies of order $1 \times$ $10^{-4} \mathrm{~s}^{-1}$ are sufficient to account for the PV anomalies seen in Fig. 13. As in the case of the Talas PV dipoles, potential temperature profiles (not shown) in the lower stratosphere through the low-PV (blue) and high-PV 


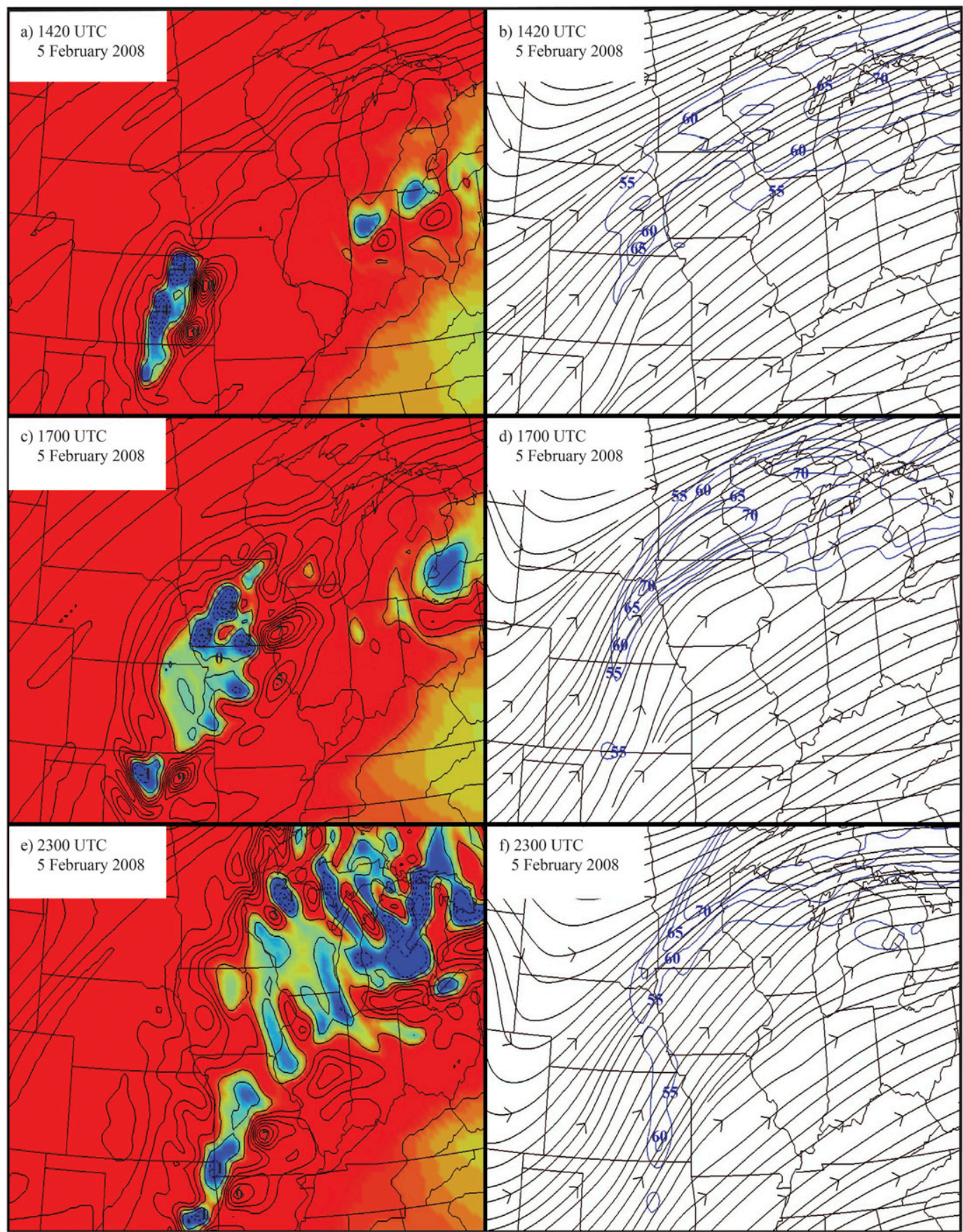

FIG. 14. Evolution of EPV and 11-km streamfunction at (a),(b) 1420, (c),(d) 1700, and (e),(f) 2300 UTC 5 Feb 2008. (left) EPV is shown in color (blue negative), with black contours every 1 PVU (dashed negative). (right) Wind speed at $9 \mathrm{~km}$ is also shown (blue contours every $5 \mathrm{~m} \mathrm{~s}^{-1}$ in the interval $55-65 \mathrm{~m} \mathrm{~s}^{-1}$ ).

(green) anomaly are distinct. In comparing the tropical and midlatitude convective cases, since midlatitude thunderstorms are not as deep, one might expect more moderate updraft maxima, with consequently reduced rates of production of $\mathrm{PV}$ anomalies and of the strength of the resulting PV maxima.
Figures $13 \mathrm{~b}$ and $13 \mathrm{~d}$ show comparisons of profiles of EPV (black), absolute vorticity (brown), and absolute vorticity scaled by density (gray) through the positive (Fig. 13b) and negative (Fig. 13d) anomalies, similar to Fig. 6 for the Talas dipole. EPV and PV are again near zero through the depth of the convection. Absolute 
vorticity is cyclonic throughout the depth of the troposphere and peaks in the positive UTLS anomaly (Fig. 13b). However, ascending below the negative anomaly, it gradually becomes anticyclonic in the upper troposphere before maximizing in the lower stratosphere. This difference for the absolute vorticity profiles below the positive and negative maxima is similar to that found for the Talas dipole (Fig. 6). Again, absolute vorticity scaled by density causes a downward diminution relative to absolute vorticity in both profiles. The agreement between PV and absolute vorticity in the PV dipole maxima reflects the fact that static stability is strongly positive entering the base of the stratosphere. Numerical differences between PV and absolute vorticity and density-scaled absolute vorticity are larger than in Fig. 6, because at 11-km density is twice as large as at $16.5 \mathrm{~km}$, so using the same scaling factor causes a factorof-2 difference here. The production of large negative and positive anomalies evidently occurs abruptly as the updraft impinges on the base of the stratosphere.

The evolution of convective PV dipoles and streamfunction at $11 \mathrm{~km}$ from 1420 to 2300 UTC 5 February 2008 is shown in Fig. 14. At 1420 UTC, the PV dipole (Fig. 14a) is found to be oriented such that the negative anomaly is to the left of the local synoptic wind (Fig. 14b).

As the convective outbreak spreads into a mesoscale convective complex (Fig. 14c), the 11-km streamfunction pattern exhibits a more divergent aspect over Iowa (cf. Figs. 14b,d). As described in Rowe and Hitchman (2016), the inertially unstable divergent outflow from this thunderstorm complex led to an increased poleward flow and jet enhancement, as seen in Fig. 14d (blue contours). By 2300 UTC, this convective activity generated a strong field of gravity waves propagating upstream, with a resulting "fishbone pattern" in EPV (Fig. 14e) and a "jet suturing" pattern between the subpolar and subtropical jets in the UTLS (Rowe and Hitchman 2015). Note the further effect of the inertially unstable outflow on the jet structure at $9 \mathrm{~km}$ seen in Fig. 14f, where the Coriolis torque on enhance poleward flow leads to an enhanced zonal jet at a higher latitude (Rowe and Hitchman 2016).

As in the case of Talas, the convective complex has significantly altered the synoptic-scale jet structure. In this case, convection occurs in an elongated trough instead of the similarly elongated anticyclonic structure in the UTLS environment of Talas. In the midlatitude case, the horizontally varying northeastward flow $(\partial v / \partial x>0)$ stretches the quasi-symmetric negative PV structures into a long PV streamer, which lies just on the equatorward side of the subpolar jet [cf. Fig. 6 of Rowe and Hitchman (2015) and Fig. 6 of Rowe and Hitchman (2016)]. This is similar to the evolution of PV structures in the extratropical transition of Talas, where $\partial v / \partial x<0$.

\section{Conclusions}

These results support the idea that there are fundamental physical processes operating in both tropical and midlatitude storms that can explain the appearance of theta and PV dipole structures in the UTLS over regions of deep convection. Ambient flow over convective updrafts can generate a negative-positive potential temperature dipole aligned with the flow. In both cases the ambient upper-tropospheric wind shear is related to the spatial orientation of PV dipoles in the UTLS, with "low PV to the left of the wind shear." In both cases, the synoptic-scale horizontal vorticity was created by ambient baroclinicity at the cyclone scale. The fact that the negative member usually lies to the left of the ambient wind shear lends strong support for the tilting/twisting mechanism. This implies that the axes of the potential temperature and PV dipoles are perpendicular to each other. In tropical cyclones the low-PV anomaly lies radially outward. This rule also abides in the Southern Hemisphere, except the positive PV element is the inertially unstable member, as found in simulations of Tropical Cyclone Ita near northeastern Australia (not shown).

Quantitative estimates of the rate of production of $\mathrm{PV}$ and diagnosis of the strength of PV anomalies in the UTLS are consistent with divergent mesoscale outflow around the PV anomalies. The tilting/twisting mechanism appears to be manifested in the upper troposphere and amplified as the updraft compresses vertically into the base of the stratosphere. The role of the tropopause seems to be crucial in the UWNMS, since PV dipoles are not observed unless convection reached the tropopause.

The existence of a synoptic-scale deformation field in the UTLS, whether cyclonic (Super Tuesday) or anticyclonic (Talas), generated a very similar and striking elongation of a pair of PV streamers, which modified the adjacent westerly jet structure. It may be of some utility with respect to mesoscale weather forecasting to further investigate the role of the inertially unstable member of PV dipoles in modifying the evolution of both extratropical and tropical cyclones.

Acknowledgments. We are grateful for support from NSF Grants AGS-1256215 and AGS-1555851. We thank Greg Tripoli and Leslie Smith for the insightful conversations and Pete Pokrandt for help with the UWNMS and data acquisition.

\section{REFERENCES}

Agusti-Panareda, A., C. D. Thorncroft, G. C. Craig, and S. L. Gray, 2004: The extratropical transition of Hurricane Irene (1999): A potential-vorticity perspective. Quart. J. Roy. Meteor. Soc., 130, 1047-1074, doi:10.1256/qj.02.140. 
Andrews, D. G., J. R. Holton, and C. B. Leovy, 1987: Middle Atmosphere Dynamics. Academic Press, 489 pp.

Bjerknes, V., 1898: Über einen hydrodynamischen fundamentalsatz und seine anwendung besonders auf die mechanik der atmosphäre und des weltmeeres. K. Sven. Vetensk. Akad. Handl., 31, 1-35.

Boer, G. J., and M. Lazare, 1988: Some results concerning the effect of horizontal resolution and gravity wave drag on simulated climate. J. Climate, 1, 789-806, doi:10.1175/ 1520-0442(1988)001<0789:SRCTEO > 2.0.CO;2.

Chagnon, J. M., and S. L. Gray, 2009: Horizontal potential vorticity dipoles on the convective storm scale. Quart. J. Roy. Meteor. Soc., 135, 1392-1408, doi:10.1002/qj.468.

—_ _ - and J. Methven, 2013: Diabatic processes modifying potential vorticity in a North Atlantic cyclone. Quart. J. Roy. Meteor. Soc., 139, 1270-1282, doi:10.1002/qj.2037.

Conzemius, R. J., and M. T. Montgomery, 2009: Clarification on the generation of absolute and potential vorticity in mesoscale convective vortices. Atmos. Chem. Phys., 9, 7591-7605, doi:10.5194/acp-9-7591-2009.

Deng, Q., L. M. Smith, and A. J. Majda, 2012: Tropical cyclogenesis and vertical shear in a moist Boussinesq model. J. Fluid Mech., 706, 384-412, doi:10.1017/jfm.2012.260.

DiMego, G. J., and L. Bosart, 1982: The transformation of Tropical Storm Agnes into an extratropical cyclone. Part II: Moisture, vorticity and kinetic energy budgets. Mon. Wea. Rev., 110, 412-433, doi:10.1175/1520-0493(1982)110<0412: TTOTSA $>2.0 . \mathrm{CO} ; 2$.

Eady, E. T., 1949: Long waves and cyclone waves. Tellus, 1, 33-52, doi:10.3402/tellusa.v1i3.8507.

Eliassen, A., and E. Kleinschmidt, 1957: Dynamic Meteorology. Handbuch der Physik, Vol. 10, Springer-Verlag, 154 pp.

Foley, G. R., and B. N. Hanstrum, 1994: The capture of tropical cyclones by cold fronts off the west coast of Australia. Wea. Forecasting, 9, 577-592, doi:10.1175/1520-0434(1994)009<0577: TCOTCB $>2.0 . \mathrm{CO} ; 2$.

Hamilton, K., and W. Ohfuchi, Eds., 2008: High Resolution Numerical Modeling of the Atmosphere and Ocean. Springer, 293 pp.

Hernandez-Duenas, G., L. M. Smith, and S. N. Stechmann, 2014: Investigation of Boussinesq dynamics using intermediate models based on wave-vertical interactions. J. Fluid Mech., 747, 247-287, doi:10.1017/jfm.2014.138.

Holton, J. R., 2006: An Introduction to Dynamic Meteorology. Academic Press, 535 pp.

Hoskins, B. J., M. E. McIntyre, and A. W. Robertson, 1985: On the use and significance of isentropic potential vorticity maps. Quart. J. Roy. Meteor. Soc., 111, 877-946, doi:10.1002/ qj. 49711147002 .

Japan Meteorological Agency, 2011: Annual report on the activities of the RSMC Tokyo-Typoon Center 2011. Japan Meteorological Agency Tech. Rep. 82 pp. [Available online at http://www.jma.go.jp/jma/jma-eng/jma-center/rsmc-hp-pub-eg/ AnnualReport/2011/Text/Text2011.pdf.]

Jascourt, S., 1997: Convective organizing and upscale development processes explored through idealized numerical experiments. Ph.D. dissertation, University of Wisconsin-Madison, $267 \mathrm{pp}$

Jones, S. C., and Coauthors, 2003: The extratropical transition of tropical cyclones: Forecast challenges, current understanding, and future directions. Wea. Forecasting, 18, 1052-1092, doi:10.1175/1520-0434(2003)018<1052:TETOTC >2.0.CO;2.

Kilroy, G., R. K. Smith, and U. Wissmeier, 2014: Tropical convection: The effects of ambient vertical and horizontal vor- ticity. Quart. J. Roy. Meteor. Soc., 140, 1756-1770, doi:10.1002/ qj. 2261.

Kitabatake, N., 2008: Extratropical transition of tropical cyclones in the western North Pacific: Their frontal evolution. Mon. Wea. Rev., 136, 2066-2090, doi:10.1175/2007MWR1958.1.

—, S. Hoshino, K. Bessho, and F. Fujibe, 2007: Structure and intensity change of Typhoon Songda (0418) undergoing extratropical transition. Pap. Meteor. Geophys., 58, 135-153, doi:10.2467/mripapers.58.135.

Klein, P. M., P. A. Harr, and R. L. Elsberry, 2000: Extratropical transition of western North Pacific tropical cyclones: An overview and conceptual model of the transforming stage. Wea. Forecasting, 15, 373-396, doi:10.1175/1520-0434(2000)015<0373: ETOWNP $>2.0 . \mathrm{CO} ; 2$.

Kleinschmidt, E., 1950a: Über aufbau und entstehung von zyklonen I (On the structure and formation of cyclones I). Meteor. Rundsch., 3, 1-6.

__ 1950b: Über aufbau and entstehung von zyklonen II (On the structure and formation of cyclones II). Meteor. Rundsch., 4, 89-96. , 1955: Die entsehung einer höhenzyklone über Nordamerika. Tellus, 7, 96-110, doi:10.3402/tellusa.v7i1.8766.

Lindzen, R. S., and M. Fox-Rabinovitz, 1989: Consistent horizontal and vertical resolution. Mon. Wea. Rev., 117, 2575-2583, doi:10.1175/1520-0493(1989)117<2575:CVAHR > 2.0.CO;2.

Mahlman, J. D., and L. J. Umscheid, 1987: Comprehensive modeling of the middle atmosphere: The influence of horizontal resolution. Transport Processes in the Middle Atmosphere, G. Visconti, Ed., D. Riedel Publishing, 251-266.

Markowski, P., and Y. Richardson, 2010: Mesoscale Meteorology in Midlatitudes. John Wiley and Sons, 407 pp.

Mecikalski, J. R., and G. J. Tripoli, 1998: Inertial available kinetic energy and the dynamics of tropical plume formation. Mon. Wea. Rev., 126, 2200-2216, doi:10.1175/1520-0493(1998)126<2200: IAKEAT $>2.0 . \mathrm{CO} ; 2$.

Muraki, D., and C. Snyder, 2007: Vortex dipoles for surface quasigeostrophic models. J. Atmos. Sci., 64, 2961-2967, doi:10.1175/ JAS3958.1.

Muramatsu, T., 1985: A study on the changes of the three-dimensional structure and movement speed of the typhoon through its life time. Meteorological Research Institute Tech. Rep. 14, 117 pp.

Oku, Y., J. Yoshino, T. Takemi, and H. Ishikawa, 2014: Assessment of heavy rainfall-induced disaster potential based on an ensemble simulation of Typhoon Talas (2011) with controlled track and intensity. Nat. Hazards Earth Syst. Sci., 14, 26992709, doi:10.5194/nhess-14-2699-2014.

Palmen, E., 1958: Vertical circulation and release of kinetic energy during the development of Hurricane Hazel into an extratropical storm. Tellus, 10, 1-13, doi:10.3402/ tellusa.v10i1.9222.

Plougonven, R., and F. Zhang, 2014: Internal gravity waves from atmospheric jets and fronts. Rev. Geophys., 52, 33-76, doi:10.1002/2012RG000419.

Pokrandt, P. J., G. J. Tripoli, and D. D. Houghton, 1996: Processes leading to the formation of mesoscale waves in the Midwest cyclone of 15 December 1987. Mon. Wea. Rev., 124, 2726-2752, doi:10.1175/1520-0493(1996)124<2726:PLTTFO>2.0.CO;2.

Randall, D., M. Khairoutdinov, A. Arakawa, and W. Grabowski, 2003: Breaking the cloud parameterization deadlock. Bull. Amer. Meteor. Soc., 84, 1547-1564, doi:10.1175/BAMS-84-11-1547.

Rowe, S. M., and M. H. Hitchman, 2015: On the role of inertial instability in stratosphere-troposphere exchange in midlatitude cyclones. J. Atmos. Sci., 72, 2131-2151, doi:10.1175/ JAS-D-14-0210.1. 
_ and - 2016: On the relationship between inertial instability, poleward momentum surges, and jet intensifications near midlatitude cyclones. J. Atmos. Sci., 73, 2299-2315, doi:10.1175/JAS-D-15-0183.1.

Schemm, S., H. Wernli, and L. Papritz, 2013: Warm conveyor belts in idealized moist baroclinic wave simulations. J. Atmos. Sci., 70, 627-652, doi:10.1175/JAS-D-12-0147.1.

Shapiro, L. J., and J. L. Franklin, 1995: Potential vorticity in Hurricane Gloria. Mon. Wea. Rev., 123, 1465-1475, doi:10.1175/ 1520-0493(1995)123<1465:PVIHG > 2.0.CO;2.

Snyder, C. D., D. J. Muraki, R. Plougonven, and F. Zhang, 2007: Inertia-gravity waves generated within a dipole vortex. J. Atmos. Sci., 64, 4417-4431, doi:10.1175/2007JAS2351.1.

_ , R. Plougonven, and D. J. Muiraki, 2009: Mechanisms for spontaneous gravity wave generation within a dipole vortex. J. Atmos. Sci., 66, 3464-3478, doi:10.1175/2009JAS3147.1.

Stephan, C., M. J. Alexander, and J. H. Richter, 2016: Characteristics of gravity waves from convection and implications for their parameterization in global circulation models. J. Atmos. Sci., 73, 2729-2742, doi:10.1175/JAS-D-15-0303.1.

Thorpe, A. J., 1986: Synoptic-scale disturbances with circular symmetry. Mon. Wea. Rev., 114, 1384-1389, doi:10.1175/ 1520-0493(1986)114<1384:SSDWCS $>2.0 . C O ; 2$.

Tripoli, G. J., 1992: A nonhydrostatic mesoscale model designed to simulate scale interaction. Mon. Wea. Rev., 120, 1342-1359, doi:10.1175/1520-0493(1992)120<1342:ANMMDT>2.0.CO;2.

Wang, P. K., 2004: A cloud model of jumping cirrus above storm top. Geophys. Res. Lett., 31, L18106, doi:10.1029/2004GL020787.
Wang, S., F. Zhang, and C. Snyder, 2009: Generation and propagation of gravity waves from jets within vortex dipoles. J. Atmos. Sci., 66, 1294-1314, doi:10.1175/2008JAS2830.1.

$\longrightarrow$, - and C. C. Epifanio, 2010: Forced gravity wave responses near the jet exit region in a linear model. Quart. J. Roy. Meteor. Soc., 136, 1773-1787, doi:10.1002/qj.676.

Wang, Y., and G. J. Holland, 1996: The beta drift of baroclinic vortices. Part I: Adiabatic vortices. J. Atmos. Sci., 53, 411-427, doi:10.1175/1520-0469(1996)053<0411:TBDOBV>2.0.CO;2.

Weijenborg, C., P. Friedrichs, and A. Hense, 2015: Organisation of potential vorticity on the mesoscale during deep moist convection. Tellus, 67A, 25705, doi:10.3402/tellusa.v67.25705.

Wu, C. C., and K. A. Emanuel, 1995: Potential vorticity diagnosis of hurricane movement. Part II: Tropical Storm Ana (1991) and Hurricane Andrew (1992). Mon. Wea. Rev., 123, 93-109, doi:10.1175/1520-0493(1995)123<0093:PVDOHM>2.0.CO;2.

Yamada, M., Y. Matsushi, M. Chigira, and J. Mori, 2012: Seismic recordings of landslides caused by Typhoon Talas (2011), Japan. Geophys. Res. Lett., 39, L13301, doi:10.1029/ 2012GL052174.

Yamada, Y., T. Sampe, Y. O. Takahashi, M. K. Yoshioka, W. Ohfuchi, M. Ishiwatari, K. Nakajima, and Y.-Y. Hayashi, 2005: A resolution dependence of equatorial precipitation activities represented in a general circulation model. Theor. Appl. Mech. Japan, 54, 289-297, doi:10.11345/nctam.54.289.

Zhang, F., and D. Tao, 2013: Effects of vertical wind shear on the predictability of tropical cyclones. J. Atmos. Sci., 70, 975-983, doi:10.1175/JAS-D-12-0133.1. 